\title{
Rational deformations of conformal mechanics
}

\author{
José F. Cariñena, ${ }^{1, *}$ Luis Inzunza, ${ }^{2, \dagger}$ and Mikhail S. Plyushchay ${ }^{2, *}$ \\ ${ }^{1}$ Departamento de Física Teórica, Universidad de Zaragoza, 50009 Zaragoza, Spain \\ ${ }^{2}$ Departamento de Física, Universidad de Santiago de Chile, Casilla 307, Santiago 2, Chile
}

(Received 1 June 2018; published 12 July 2018)

\begin{abstract}
We study deformations of the quantum conformal mechanics of de Alfaro-Fubini-Furlan with a rational additional potential term generated by applying the generalized Darboux-Crum-Krein-Adler transformations to the quantum harmonic oscillator and by using the method of dual schemes and mirror diagrams. In this way we obtain infinite families of isospectral and nonisospectral deformations of the conformal mechanics model with special values of the coupling constant $g=m(m+1), m \in \mathbb{N}$, in the inverse square potential term, and for each completely isospectral or gapped deformation given by a mirror diagram, we identify the sets of the spectrum-generating ladder operators that encode and coherently reflect its fine spectral structure. Each pair of these operators generates a nonlinear deformation of the conformal symmetry, and their complete sets pave the way for investigation of the associated superconformal symmetry deformations.
\end{abstract}

DOI: $10.1103 /$ PhysRevD.98.026017

\section{INTRODUCTION}

Conformal mechanics of de Alfaro, Fubini, and Furlan (AFF) was introduced and studied as a $(0+1)$-dimensional conformal field theory [1]. This system corresponds to the simplest two-body case of the $N$-body Calogero model [2-4] with eliminated center of mass degree of freedom. The same quantum harmonic oscillator model with a centrifugal barrier [1] appeared in the literature later under the name of the isotonic oscillator [5]. It found interesting applications, particularly in the context of the physics of anyons [6-8]. For some references devoted to the investigation of different aspects of this model and its generalizations see [9-16]. Some time ago a new wave of interest to this model and its supersymmetric generalizations appeared in the context of the AdS/CFT correspondence [17-19] after the observation that the dynamics of a particle near the horizon of the extreme Reissner-Nordström black hole is governed by some relativistic type of conformal mechanics [20-28]. Recently, the model also attracted attention in connection with the confinement problem in QCD [29,30]. The isotonic oscillator also caused a notable interest in investigations related to exceptional orthogonal polynomials [31-38].

The method of the Darboux-Crum-Krein-Adler (DCKA) transformations [39-43] provides a simple mechanism for the construction of the rational extensions of the quantum harmonic oscillator (described by the potential

\footnotetext{
*jfc@unizar.es

luis.inzunza@usach.cl

*mikhail.plyushchay@usach.cl
}

that is composed from the quadratic term and additionally generated rational term) with any preassigned almost equidistant gapped spectrum containing an arbitrary finite even number of missing energy levels in it $[43,44]$. The same method recently allowed one to identify the set of the ladder operators for rationally extended quantum harmonic oscillator systems that detect and coherently reflect all their spectral peculiarities and form the sets of the spectrumgenerating operators [45].

(i) In the present paper, we address the problem of construction of the complete sets of the spectrumgenerating ladder operators for rational deformations of the conformal mechanics characterized by special values of the coupling constant $g=m(m+1)$, $m \in \mathbb{N}$, in the inverse square potential term. Each pair of such ladder operators provides us with a certain nonlinear deformation of the conformal symmetry, and their complete sets pave the way for investigation of the associated superconformal symmetry deformations.

The conformal mechanics model with the indicated values of the coupling constant in the inverse square potential term is especially interesting from the point of view of applications to the physics of anyons where the cases of $g=m(m+1)$ describe the relative dynamics of the bosons $[6-8,46]$. Another reason is that the potentials $m(m+1) / x^{2}$ without the confining harmonic term are related to the hierarchy of the Korteweg-de Vries (KdV) equation within a framework of a broader picture in which the Calogero systems govern the dynamics of the moving poles of the rational solutions to the $\mathrm{KdV}$ equation [47-49]. 
We start from a certain set of physical and nonphysical eigenstates of the quantum harmonic oscillator selected as the seed states for the DCKA transformations, and we obtain infinite families of rational extensions of the isotonic oscillators for both isospectral and nonisospectral cases with an arbitrary (odd or even) number of missing energy levels in the gaps. For this, we develop and apply the method of the mirror diagrams allowing us to identify complementary sets of the seed states that produce the same system modulo a relative displacement of the spectrum. Having two dual schemes for the same system, we construct the sets of the ladder operators that generate nonlinearly deformed conformal symmetry and coherently reflect all the spectral properties of each isospectral and nonisospectral deformation.

The paper is organized as follows. The next section is devoted to the construction and analysis of the quantum conformal mechanics systems by applying the DarbouxCrum (DC) transformations to the half-harmonic oscillator. One of the two dual families of transformations we use is based on the selection of physical eigenstates of the quantum harmonic oscillator as the seed states. Another family of Darboux transformations employs nonphysical eigenstates generated from physical eigenfunctions by a spatial Wick rotation. The same two types of the Darboux transformations are employed for the construction of the ladder operators of the isotonic oscillator systems. We also observe there how ladder operators can be generated from the two supersymmetric schemes corresponding to the broken and unbroken phases. In Sec. III we describe the construction of the dual schemes of the DCKA transformations by generalizing the relations from Sec. II. We show there that the dual schemes can conveniently be presented by a mirror diagram, in which they are related by a kind of a "charge conjugation." The dual schemes and mirror diagrams lie in the basis of constructions of the next two sections. In Sec. IV we construct infinite families of the completely isospectral rational deformations of the conformal mechanics systems and describe their ladder operators. In Sec. V we describe the construction of rational deformations with any preassigned gapped spectrum organized in an arbitrary number of the lower-lying "valence" bands of arbitrary size and infinite equidistant band. We also consider there the construction of the pairs of ladder operators that reflect different properties of the spectrum, and then we identify their combinations that form the sets of the spectrum-generating operators. Each such pair of the ladder operators generates a certain nonlinear deformation of the conformal symmetry. In Sec. VI we summarize and discuss the results, as well as indicate some problems that could be interesting for further investigation.

\section{QUANTUM CONFORMAL MECHANICS SYSTEMS}

Consider the quantum harmonic oscillator system described by the Hamiltonian defined by the differential operator $L^{\text {osc }}=-\frac{d^{2}}{d x^{2}}+x^{2}$ with domain $L^{2}(\mathbb{R}, d x)$. The solutions to the spectral problem $L^{\mathrm{osc}} \psi_{n}=E_{n} \psi_{n}$ are given by the normalizable eigenfunctions $\psi_{n}(x)=H_{n}(x) e^{-x^{2} / 2}$ corresponding to the energy values $E_{n}=2 n+1, n=0$, $1, \ldots$, where $H_{n}(x)$ are the Hermite polynomials. Here and in what follows we do not preoccupy ourselves with normalization of the states and specify wave functions modulo multiplication by a nonzero complex number. A simple change $x \rightarrow i x$ (see $[31,33,38,44,50]$ ) provides us with nonphysical (non-normalizable) solutions $\psi_{n}^{-}(x)=$ $\mathcal{H}_{n}(x) e^{x^{2} / 2}$ that correspond to eigenvalues $E_{n}^{-}=-E_{n}$, where $\mathcal{H}_{n}(x)=(-i)^{n} H_{n}(i x)$. These not physically acceptable states will play an important role in the construction of the rational deformations of the quantum mechanics systems.

The confining AFF model [1] is characterized by the $\mathfrak{s} \mathfrak{l}(2, \mathbb{R})$ conformal symmetry

$$
\left[L_{g}^{\text {iso }}, \mathcal{C}_{g}^{ \pm}\right]= \pm 4 \mathcal{C}_{g}^{ \pm}, \quad\left[\mathcal{C}_{g}^{-}, \mathcal{C}_{g}^{+}\right]=8 L_{g}^{\text {iso }},
$$

where $L_{g}^{\text {iso }}$ is the Hamiltonian given by a Schrödinger-type differential operator

$$
L_{g}^{\text {iso }}=-\frac{d^{2}}{d x^{2}}+x^{2}+\frac{g}{x^{2}}, \quad g \in \mathbb{R},
$$

defined in a domain $\left\{\phi \in L^{2}((0, \infty), d x) \mid \phi\left(0^{+}\right)=0\right\}$, and $\mathcal{C}_{g}^{ \pm}$are the spectrum-generating ladder operators that we specify below. When $g \geq-1 / 4$, one can replace $g=$ $\nu(\nu+1)$ with $\nu \in \mathbb{R}$. For $g>-\frac{1}{4}$, the factorization can be done in two ways because $g(\nu)=g(-(1+\nu))$. We are interested here in the case when $\nu$ takes integer values and write

$L_{m}^{\text {iso }}=-\frac{d^{2}}{d x^{2}}+x^{2}+\frac{m(m+1)}{x^{2}}, \quad m=0,1, \ldots$

The first member $L_{0}^{\text {iso }}$ of this infinite family corresponds to a half-harmonic oscillator (on the half-line $x>0$ ) with a nonpenetrable infinite barrier put at $x=0$, i.e., to the quantum system given by the potential $V(x)=+\infty$ for $x \leq 0$ and $V(x)=x^{2}$ for $x>0$. Its physical eigenstates $\psi_{0, l}$ and eigenvalues $E_{0, l}=4 l+3, l=0,1, \ldots$, correspond to those of the quantum harmonic oscillator with odd index $n=2 l+1: \quad \psi_{0, l}(x)=\psi_{2 l+1}(x)$, for $x \in(0, \infty)$, $E_{0, l}=E_{2 l+1}$. Hereafter with an abuse of notation we write that two functions with a different domain are equal when both coincide on the common domain, and in the preceding case we simply write $\psi_{0, l}=\psi_{2 l+1}$.

The eigenstates and eigenvalues of the isotonic (radial) oscillator $L_{m}^{\text {iso }}$ with $m \geq 1$ can be found, particularly, by applying the DC transformations to the half-harmonic oscillator system $L_{0}^{\text {iso }} \equiv L_{0}$. Taking the first $m$ eigenfunctions $\psi_{0,0}, \ldots, \psi_{0, m-1}, m=1,2, \ldots$, of $L_{0}$ as the seed states 
for the DC transformation (see Appendix A), we generate the quantum Hamiltonian

$$
\begin{aligned}
L_{m} & \equiv-\frac{d^{2}}{d x^{2}}+x^{2}-2(\ln W(1,3, \ldots, 2 m-1))^{\prime \prime} \\
& =L_{m}^{\text {iso }}+2 m .
\end{aligned}
$$

Here and in what follows we use a compact notation $n=\psi_{n}(x),-n=\psi_{n}^{-}(x), \tilde{n}=\widetilde{\psi_{n}(x)}, \widetilde{-n}=\widetilde{\psi_{n}^{-}(x)}$ for the corresponding physical and nonphysical eigenstates of the quantum harmonic oscillator, where $\widetilde{\psi_{n}(x)}$ is an eigenfunction linearly independent from a solution $\psi_{n}(x)$ of the corresponding stationary Schrödinger equation; see Eq. (A3). According to this notation, $W(1,3, \ldots, 2 m-1)$ is the Wronskian of the odd eigenfunctions $\psi_{1}=\psi_{0,0}$, $\psi_{3}=\psi_{0,1}, \ldots, \psi_{2 m-1}=\psi_{0, m-1}$ of the quantum harmonic oscillator. Hereafter we identify an eigenfunction of differential operators $L$ as a physical eigenstate of the corresponding Hamiltonian $H$ when it satisfies the integrability and boundary conditions. Accordingly, a nonphysical eigenstate is not a true eigenstate of the Hamiltonian operator.

Given the physical character of the eigenstates of $L_{0}=L_{0}^{\text {iso }}$ that we used as seed states for the DC transformation (2.4), the corresponding energy levels are absent in the spectrum of the generated system $L_{m}$. The neighbor members $L_{m-1}$ and $L_{m}$ of the family of the differential operators and their corresponding Hamiltonians are factorized and intertwined by the first order operators

$$
A_{m}^{-}=\frac{d}{d x}+x-\frac{m}{x}, \quad A_{m}^{+}=-\frac{d}{d x}+x-\frac{m}{x},
$$

in terms of which we have

$$
\begin{array}{r}
A_{m}^{+} A_{m}^{-}=L_{m-1}-E_{2 m-1}, \quad A_{m}^{-} A_{m}^{+}=L_{m}-E_{2 m-1}, \\
A_{m}^{-} L_{m-1}=L_{m} A_{m}^{-}, \quad A_{m}^{+} L_{m}=L_{m-1} A_{m}^{+} .
\end{array}
$$

Here $E_{2 m-1}=4 m-1=E_{m-1,0}$ corresponds to the eigenvalue of the eigenfunction $\psi_{m-1,0}=x^{m} e^{-x^{2} / 2}$ that describes the ground state of the system $L_{m-1}$. According to Eq. (A1), the state $\psi_{*}=\psi_{m-1,0}$ generates the operators (2.5). Any eigenstate $\psi_{m, E}$ of energy $E \neq E_{m-1,0}$ of the system $L_{m}$ can be generated from the state $\psi_{m-1, E}$ that is an eigenstate of $L_{m-1}$ of the same energy, $\psi_{m, E}=A_{m}^{-} \psi_{m-1, E}$. The nonphysical eigenstate $\left(\psi_{m-1,0}\right)^{-1}$ of $L_{m}$ of energy $E=E_{m-1,0}$ is generated by applying the operator $A_{m}^{-}$to the nonphysical eigenstate $\widetilde{\psi_{m-1,0}}$ of $L_{m-1}$.

The factorization and intertwining relations satisfied by operators (2.5) can be translated into the language of supersymmetric quantum mechanics by taking the $2 \times 2$ diagonal matrix

$$
\begin{aligned}
\mathcal{H}_{m}^{e} & =\operatorname{diag}\left(\mathcal{H}_{m}^{e,+} \equiv L_{m}-E_{2 m-1}, \mathcal{H}_{m}^{e,-} \equiv L_{m-1}-E_{2 m-1}\right) \\
m & =1, \ldots,
\end{aligned}
$$

as the Hamiltonian operator. The antidiagonal supercharge operators are defined by

$$
\begin{aligned}
\mathcal{Q}_{m}^{e, 1} & =\left(\begin{array}{cc}
0 & A_{m}^{-} \\
A_{m}^{+} & 0
\end{array}\right) \\
\mathcal{Q}_{m}^{e, 2} & =i \sigma_{3} \mathcal{Q}_{m}^{e, 1}=\left(\begin{array}{cc}
0 & i A_{m}^{-} \\
-i A_{m}^{+} & 0
\end{array}\right) .
\end{aligned}
$$

They generate the Lie superalgebra of $\mathcal{N}=2$ supersymmetry,

$\left[\mathcal{H}_{m}^{e}, \mathcal{Q}_{m}^{e, \alpha}\right]=0, \quad\left\{\mathcal{Q}_{m}^{e, \alpha}, \mathcal{Q}_{m}^{e, \beta}\right\}=2 \delta^{\alpha, \beta} \mathcal{H}_{m}^{e}, \quad \alpha, \beta=1,2$

The matrix Hamiltonian operator $\mathcal{H}_{m}^{e}$ has the singlet ground state $\Psi_{0, m}=\left(0, \psi_{m-1,0}\right)^{t}$ of zero energy that is annihilated by both supercharges $\mathcal{Q}_{m}^{e, \alpha}$, and so the extended system (2.8), (2.9) corresponds to the case of the exact, unbroken supersymmetry.

In correspondence with the described picture, physical eigenstates $\psi_{m, l}$, of $L_{m}$ of energies $E_{m, l}=4(m+l)+3$, $l=0,1, \ldots$, can be generated from the odd eigenstates $\psi_{2(m+l)+1}$ of the quantum harmonic oscillator of the same energies $E_{2(m+l)+1}=4(m+l)+3$,

$\psi_{m, l}=\mathbb{A}_{m}^{-} \psi_{2(m+l)+1}, \quad \mathbb{A}_{m}^{-}=A_{m}^{-} A_{m-1}^{-} \cdots A_{1}^{-}$.

In the particular case of $l=0$, relation (2.11) gives the ground state of the system $L_{m}, \psi_{m, 0}=\mathbb{A}_{m}^{-} \psi_{2 m+1}=$ $C x^{m+1} e^{-x^{2} / 2}$. The operator $\mathbb{A}_{m}^{-}$annihilates all the $m$ lowest odd eigenstates $\psi_{2 n-1}$ of the quantum harmonic oscillator with $n=1, \ldots, m$. If $\psi_{E}$ is any physical or nonphysical eigenstate of the quantum harmonic oscillator with $E \neq E_{2 n-1}=4 n-1, n=1, \ldots, m$, Eq. (2.11) can be generalized as follows:

$$
\psi_{m, E}=\mathbb{A}_{m}^{-} \psi_{E}, \quad L_{m} \psi_{m, E}=E \psi_{m, E}
$$

Here $\psi_{m, E}$ is an eigenstate of $L_{m}$ of the same, physical or nonphysical, nature excepting the case when $E=E_{2 l}$, $l=0,1, \ldots$, and $\psi_{E}=\psi_{2 l}$. The physical eigenstate $\psi_{2 l}$ of the quantum harmonic oscillator being a nonvanishing at $x=0$ even function is mapped into a nonphysical eigenstate of $L_{m}$ that is singular at $x=0$. Notice that the relation (2.12) can be presented equivalently in a Wronskian form, $\psi_{m, E}=W\left(1,3, \ldots, 2 m-1, \psi_{E}\right) / W(1,3, \ldots, 2 m-1)$. Using the factorization and intertwining relations satisfied by operators (2.5), we find the relations 
$\mathbb{A}_{m}^{+} \mathbb{A}_{m}^{-}=\prod_{j=1}^{m}\left(L_{0}-E_{2 j-1}\right), \quad \mathbb{A}_{m}^{-} \mathbb{A}_{m}^{+}=\prod_{j=1}^{m}\left(L_{m}-E_{2 j-1}\right)$,

and $\mathbb{A}_{m}^{-} L_{0}=L_{m} \mathbb{A}_{m}^{-}, \mathbb{A}_{m}^{+} L_{m}=L_{0} \mathbb{A}_{m}^{+}$for the higher order differential operators $\mathbb{A}_{m}^{-}, \mathbb{A}_{m}^{+}=\left(\mathbb{A}_{m}^{-}\right)^{\dagger}$. Equation (2.13) is useful, particularly, to establish the exact relationship between eigenfunctions (2.11) of the isotonic oscillator given in terms of the Hermite polynomials and their standard representation in terms of the generalized Laguerre polynomials; see Appendix B.

The method of dual schemes and mirror diagrams described below in Sec. III, allows us to find alternative ways to generate the isotonic oscillator system $L_{m}$ by means of the DC transformations. This can be done, particularly, by using the odd nonphysical eigenstates $\psi_{2 l+1}^{-}$of the quantum harmonic oscillator instead of its physical odd eigenstates $\psi_{2 l+1}$. The states $\psi_{2 l+1}^{-}$are also nonphysical eigenstates of the half-harmonic oscillator $L_{0}=L_{0}^{\text {iso }}$ of eigenvalues $-4 l-3$, and instead of (2.4) we obtain

$$
L_{m}-4 m=-\frac{d^{2}}{d x^{2}}+x^{2}-2 \frac{d^{2}}{d x^{2}}(\ln W(-1,-3, \ldots,-(2 m-1)))=L_{m}^{\text {iso }}-2 m
$$

We have here the alternative representation for eigenfunctions (2.12) of $L_{m}: \psi_{m, E}=W(-1,-3, \ldots,-(2 m-1)$, $\left.\psi_{E}\right) / W(-1,-3, \ldots,-(2 m-1))$. Because of a nonphysical nature of the seed states, in this case there are no removed energy values in the spectrum of (2.14) in comparison with the spectrum of the half-harmonic oscillator $L_{0}=L_{0}^{\text {iso }}$. The DC transformation of nonphysical eigenstate $\widetilde{\psi_{0, l}^{-}}=\widetilde{\psi_{2 l+1}^{-}}$ of $L_{0}, l=1, \ldots, m$, produces a nonphysical eigenstate of the Hamiltonian operator $L_{m}-4$. As a result, the system described by the differential operator $L_{m}-4$ turns out to be completely isospectral to $L_{0}$. We shall show below that this picture of DC transformations corresponds to the case of spontaneously broken supersymmetry. The relative nonzero shift $-4 m$ in (2.14) in comparison with (2.4) is important and lies in the base of the construction of the ladder operators.

The shift $-4 m$ in (2.14) is associated with the difference in the exponential factors in the structure of the physical states $\psi_{n}(x)$ of the quantum harmonic oscillator and its nonphysical eigenstates $\psi_{n}^{-}(x)=C \psi_{n}(i x)$. The structure of the Wronskian $(2.4)$ is $W(1,3, \ldots, 2 m-1)=C x^{\Delta_{m}} e^{-m x^{2} / 2}$, where $\Delta_{m}=m(m+1) / 2$ (see Appendix C), while the Wronskian in (2.14) is obtained from it by the change $x \rightarrow i x, W(-1,-3, \ldots,-(2 m-1))=C x^{\Delta_{m}} e^{m x^{2} / 2}$.

As in the case of the DC scheme based on physical eigenstates of $L_{0}$, one can factorize and intertwine the neighbor members in the family of differential operators of the isotonic oscillator systems by the first order differential operators

$A_{-m}^{-}=\frac{d}{d x}-x-\frac{m}{x}, \quad A_{-m}^{+}=-\frac{d}{d x}-x-\frac{m}{x}$.

These operators can be obtained by means of relation (A1) with $\psi_{*}=\psi_{m-1,0}^{-}$, where $\psi_{m-1,0}^{-}=\psi_{m-1,0}(i x)=x^{m} e^{x^{2} / 2}$ is a nonphysical eigenstate of $L_{m-1}$ of eigenvalue -3 . Note that the nonphysical eigenstate $\psi_{m-1,0}^{-}$of $L_{m-1}$ can be generated from the nonphysical eigenstate $\psi_{2 m+1}^{-}$of the quantum harmonic oscillator if we take there $\psi_{E}=\psi_{2 m+1}^{-}$.

If we formally put $m=0$ in $A_{m}^{ \pm}$and $A_{-m}^{ \pm}$, we obtain the ladder operators $\left(a^{ \pm}\right)$of the harmonic oscillator. They are not of the same nature as $A_{m}^{ \pm}$or $A_{-m}^{ \pm}$since they intertwine harmonic oscillators with relatively displaced spectra and transform physical states of $L_{0}$ into its nonphysical states: $a^{ \pm} \psi_{2 n+1}=\psi_{2 n+1 \pm 1}$. Nevertheless, they produce the ladder operators $\left(a^{ \pm}\right)^{2}$ of $L_{0}$, and they will be used in the construction of mirror diagrams in the next section.

We have the factorization relations $A_{-m}^{-} A_{-m}^{+}=L_{m}^{\text {iso }}+$ $2 m-1$ and $A_{-m}^{-} A_{-m}^{+}=L_{m}-1$. Because of different constants of factorization, the operators $A_{-m}^{-}$and $A_{-m}^{+}$, unlike $A_{m}^{-}$and $A_{m}^{+}$, intertwine the differential operators $L_{m-1}$ and $L_{m}$ with an additional nonzero shift,

$$
\begin{aligned}
A_{-m}^{-} L_{m-1} & =\left(L_{m}-\Delta E^{\text {iso }}\right) A_{-m}^{-}, \\
A_{-m}^{+} L_{m} & =\left(L_{m-1}+\Delta E^{\text {iso }}\right) A_{-m}^{+} .
\end{aligned}
$$

Here $\Delta E^{\text {iso }}=4$ is the difference between any two successive levels in the equidistant spectrum of $L_{m}$ for any value of $m$. As a consequence, the application of $A_{-m}^{-}$to a physical or nonphysical eigenstate $\psi_{m-1, E}$ of $L_{m-1}$ of eigenvalue $E \neq-3, L_{m-1} \psi_{m-1, E}=E \psi_{m-1, E}$, produces a state $A_{-m}^{-} \psi_{m-1, E}$ that is an eigenstate of $L_{m}$ of eigenvalue $E+4$ having the same physical or nonphysical nature. Similarly, the application of $A_{-m}^{+}$to an eigenstate $\psi_{m, E}$ of $L_{m}$ of eigenvalue $E \neq+1$ produces the state $A_{-m}^{+} \psi_{m, E}$ that is an eigenstate of $L_{m-1}$ of eigenvalue $E-4$ having the same, physical or nonphysical, nature as the state $\psi_{m, E}$. Besides, in accordance with Eq. (A3), the application of $A_{m}^{-}$ and $A_{m}^{+}$to the partner states $\tilde{\psi}$ of the kernels of $A_{m}^{+}$and $A_{m}^{-}$ reproduces the corresponding nonphysical eigenstates of $L_{m}$ and $L_{m-1}$ of energies $E=+1$ and -3 , respectively.

Analogously to (2.8) and (2.9), we can translate the factorization and intertwining relations satisfied by the operators $A_{-m}^{-}$and $A_{-m}^{+}$into the language of supersymmetric 
quantum mechanics by defining the extended Hamiltonian and supercharge operators as follows:

$$
\begin{gathered}
\mathcal{H}_{m}^{b}=\operatorname{diag}\left(\mathcal{H}_{m}^{b,+} \equiv L_{m}-1, \mathcal{H}_{m}^{b,-} \equiv L_{m-1}+3\right) \\
m=1, \ldots \\
\mathcal{Q}_{m}^{b, 1}=\left(\begin{array}{cc}
0 & A_{-m}^{-} \\
A_{-m}^{+} & 0
\end{array}\right) \\
\mathcal{Q}_{m}^{b, 2}=i \sigma_{3} \mathcal{Q}_{m}^{b, 1}=\left(\begin{array}{cc}
0 & i A_{-m}^{-} \\
-i A_{-m}^{+} & 0
\end{array}\right) .
\end{gathered}
$$

They generate, again, the Lie superalgebra of $\mathcal{N}=2$ supersymmetry,

$$
\left[\mathcal{H}_{m}^{b}, \mathcal{Q}_{m}^{b, \alpha}\right]=0, \quad\left\{\mathcal{Q}_{m}^{b, \alpha}, \mathcal{Q}_{m}^{b, \beta}\right\}=2 \delta^{\alpha, \beta} \mathcal{H}_{m}^{b}, \quad \alpha, \beta=1,2
$$

But here the lowest energy eigenvalue of the extended Hamiltonian operator $\mathcal{H}_{m}^{b}$ is positive, $E_{0, m}^{b}=4 m+2 \geq 6$, and doubly degenerate as at any higher energy level. The corresponding eigenstates are $\Psi_{0, m}^{+}=\left(\psi_{m, 0}, 0\right)^{t}$ and $\Psi_{0, m}^{-}=\left(0, \psi_{m-1,0}\right)^{t}$. Any linear combination of the states $\Psi_{0, m}^{+}$and $\Psi_{0, m}^{-}$is not annihilated by the supercharges $\mathcal{Q}_{m}^{b, \alpha}$, and the system (2.17), (2.18) corresponds to the case of the broken supersymmetry. The difference between extended Hamiltonians (2.17) and (2.8) is given by the operator $\mathcal{H}_{m}^{b}-\mathcal{L}_{m}^{e}=E_{2 m-1}+1-2 \sigma_{3} \equiv \mathcal{I}_{m}$, that is, an even integral for both supersymmetric systems $\mathcal{H}_{m}^{e}$ and $\mathcal{H}_{m}^{b}$. This integral does not commute with the supercharges but generates a rotation in index $\alpha:\left[\mathcal{I}_{m}, \mathcal{Q}_{m}^{e(b), \alpha}\right]=4 i \epsilon^{\alpha \beta} \mathcal{Q}_{m}^{e(b), \beta}$.

Similar to what we did in (2.11), one can construct differential operators of order $m, \quad \mathbb{A}_{-m}^{-}=A_{-m}^{-} \ldots A_{-1}^{-}$. Unlike the operators $\mathbb{A}_{m}^{-}$and $\mathbb{A}_{m}^{+}$, they intertwine the system $L_{m}$ with the half-harmonic oscillator $L_{0}$ with an additional shift that depends on $m, \mathbb{A}_{-m}^{-} L_{0}=$ $\left(L_{m}-4 m\right) \mathbb{A}_{-m}^{-}, \mathbb{A}_{-m}^{+} L_{m}=\left(L_{0}+4 m\right) \mathbb{A}_{-m}^{+}$. This shift also appears in the relations

$$
\begin{aligned}
& \mathbb{A}_{-m}^{+} \mathbb{A}_{-m}^{-}=\prod_{j=1}^{m}\left(L_{0}+E_{2 j-1}\right), \\
& \mathbb{A}_{-m}^{-} \mathbb{A}_{-m}^{+}=\prod_{j=1}^{m}\left(L_{m}+E_{2 j-1}-4 m\right),
\end{aligned}
$$

and $L_{m} \psi_{m, E+4 m}=(E+4 m) \psi_{m, E+4 m}$, where $\psi_{m, E+4 m}=$ $\mathbb{A}_{-m}^{-} \psi_{E}$.

Because of the shift in the intertwining relations generated by $A_{-m}^{-}$and $A_{-m}^{+}$in comparison with those for $A_{m}^{-}$ and $A_{m}^{+}$, the appropriate products of the operators from both pairs can be used to generate the second order ladder operators for the isotonic oscillator system $L_{m}^{\text {iso }}$. These are

$$
\begin{aligned}
& A_{m}^{-} A_{-m}^{+}=-\left(a^{-}\right)^{2}+\frac{m(m+1)}{x^{2}} \equiv \mathcal{C}_{m}^{-}, \\
& A_{-m}^{-} A_{m}^{+}=-\left(a^{+}\right)^{2}+\frac{m(m+1)}{x^{2}} \equiv \mathcal{C}_{m}^{+},
\end{aligned}
$$

where $a^{-}=\frac{d}{d x}+x$ and $a^{+}=-\frac{d}{d x}+x$. The products of the same operators but with the changed order are the ladder operators for the system $L_{m-1}^{\text {iso }}$,

$A_{-m}^{+} A_{m}^{-}=-\left(a^{-}\right)^{2}+\frac{m(m-1)}{x^{2}}=\mathcal{C}_{m-1}^{-}$,

$A_{m}^{+} A_{-m}^{-}=-\left(a^{+}\right)^{2}+\frac{m(m-1)}{x^{2}}=\mathcal{C}_{m-1}^{+}$.

Notice here that the products of the supercharges of the different extended systems (2.8) and (2.17) generate the ladder operators for the corresponding isotonic oscillator subsystems: $\mathcal{Q}_{m}^{e, 1} \mathcal{Q}_{m}^{b, 1}=\operatorname{diag}\left(\mathcal{C}_{m}^{-}, \mathcal{C}_{m-1}^{+}\right), \mathcal{Q}_{m}^{b, 1} \mathcal{Q}_{m}^{e, 1}=$ $\operatorname{diag}\left(\mathcal{C}_{m}^{+}, \mathcal{C}_{m-1}^{-}\right)$.

In correspondence with the distance $\Delta E^{\text {iso }}=4$ between energy levels in the equidistant spectrum of any isotonic oscillator system $L_{m}^{\text {iso }}$, the ladder operators $\mathcal{C}_{m}^{+}$and $\mathcal{C}_{m}^{-}$satisfy the commutation relations $\left[L_{m}^{\text {iso }}, \mathcal{C}_{m}^{ \pm}\right]= \pm 4 \mathcal{C}_{m}^{ \pm}$, $\left[\mathcal{C}_{m}^{-}, \mathcal{C}_{m}^{+}\right]=8 L_{m}^{\text {iso }}$ of generators of the $\mathfrak{s l}(2, \mathbb{R})$ conformal symmetry (2.1). The kernel of the lowering ladder operator is

$$
\operatorname{ker} \mathcal{C}_{m}^{-}=\operatorname{span}\left\{\left(\psi_{m-1,0}^{-}\right)^{-1}, \psi_{m, 0}\right\},
$$

where $\left(\psi_{m-1,0}^{-}\right)^{-1}$ is the nonphysical eigenstate of $L_{m}^{\text {iso }}$ of eigenvalue $E=-2 m+1$, and $\psi_{m, 0}$ is the ground state of $L_{m}^{\text {iso }}$ of energy $E_{m, 0}^{\text {iso }}=2 m+3$. The kernel of the raising operator $\mathcal{C}_{m}^{+}$is

$$
\operatorname{ker} \mathcal{C}_{m}^{+}=\operatorname{span}\left\{\psi_{m, 0}^{-},\left(\psi_{m-1,0}\right)^{-1}\right\},
$$

where the first and second states are nonphysical eigenstates of $L_{m}^{\text {iso }}$ of eigenvalues $E=-2 m-3$ and $E=2 m-1$, respectively.

In correspondence with the fact that the isotonic oscillator $L_{m}^{\text {iso }}$ has an equidistant spectrum like a half-harmonic oscillator $L_{0}^{\text {iso }}$, the pair $\mathcal{C}_{m}^{+}$and $\mathcal{C}_{m}^{-}$are the spectrum generating operators of $L_{m}^{\text {iso: }}$ any physical eigenstate of $L_{m}^{\text {iso }}$ can be produced from any other of its physical eigenstate by successive application of these ladder operators.

Operators $\mathcal{C}_{m-1}^{ \pm}$can be Darboux dressed by $A_{m}^{ \pm}$or $A_{-m}^{ \pm}$, but in this way we only produce the same ladder operators $\mathcal{C}_{m}^{+}$and $\mathcal{C}_{m}^{-}$multiplied by $\left(L_{m}^{\text {iso }}+\right.$ const $): A_{ \pm m}^{-} \mathcal{C}_{m-1}^{+} A_{ \pm m}^{+}=$ $\left(L_{m}^{\text {iso }} \mp(2 m-1)\right) \mathcal{C}_{m}^{+}$. Iterating this procedure, we can construct the ladder operators $\mathcal{C}_{m}^{ \pm}$by Darboux dressing the ladder operators $-\mathcal{C}_{0}^{ \pm}=\left(a^{ \pm}\right)^{2}$ of the half-harmonic oscillator $L_{0}^{\text {iso }}$. Explicitly, we have 


$$
\mathbb{A}_{m}^{-} \mathcal{C}_{0}^{+} \mathbb{A}_{m}^{+}=\mathbb{A}_{m}^{-} \mathbb{A}_{m}^{+} \mathcal{C}_{m}^{+}, \quad \mathbb{A}_{-m}^{-} \mathcal{C}_{0}^{+} \mathbb{A}_{-m}^{+}=\mathcal{C}_{m}^{+} \mathbb{A}_{-m}^{-} \mathbb{A}_{-m}^{+},
$$

where the products $\mathbb{A}_{m}^{-} \mathbb{A}_{m}^{+}$and $\mathbb{A}_{-m}^{-} \mathbb{A}_{-m}^{+}$are the polynomials of the $L_{m}^{\text {iso }}$ given by Eqs. (2.13) and (2.20).

By gluing appropriately different intertwining operators, we obtain the ladder operators $\tilde{\mathcal{C}}_{m}^{-}=\mathbb{A}_{m}^{-} \mathbb{A}_{-m}^{+}, \tilde{\mathcal{C}}_{m}^{+}=\mathbb{A}_{-m}^{-} \mathbb{A}_{m}^{+}$, for which $\left[L_{m}^{\text {iso }}, \tilde{\mathcal{C}}_{m}^{ \pm}\right]= \pm 4 m \tilde{\mathcal{C}}_{m}^{ \pm}$. These ladder operators, however, are not independent from $\mathcal{C}_{m}^{ \pm}$. Using relations (2.21) we find that they are reduced just to the degrees of the ladder operators $\mathcal{C}_{m}^{ \pm}: \tilde{\mathcal{C}}_{m}^{ \pm}=\left(\mathcal{C}_{m}^{ \pm}\right)^{m}$. According to this property, the lowering operator $\tilde{\mathcal{C}}_{m}^{-}$annihilates the $m$ lowest eigenstates of the isotonic oscillator $L_{m}^{\text {iso }}$, and over each one of these states we have an infinite tower of states, where the operators $\tilde{\mathcal{C}}_{m}^{ \pm}$act irreducibly. So, for $m>1$, the ladder operators $\tilde{\mathcal{C}}_{m}^{ \pm}$are not spectrum-generating operators, and the Hilbert space of the system $L_{m}^{\text {iso }}$ is separated into $m$ subspaces invariant under their action. Furthermore, we shall see that in the general case of the rationally extended isotonic oscillator systems one can construct the analogs of the operators $\mathcal{C}_{m}^{ \pm}$and $\tilde{\mathcal{C}}_{m}^{ \pm}$. Such analogs will reproduce some of the described properties of the operators $\mathcal{C}_{m}^{ \pm}$and $\tilde{\mathcal{C}}_{m}^{ \pm}$, but the general picture related to ladder operators will be more complicated there.

In conclusion of this section we note that the Hamiltonian operator $L_{m}^{\text {iso }}$ is invariant under the change $m \rightarrow-(m+1)$. If we apply this transformation to the intertwining operators, we obtain $A_{m}^{-} \leftrightarrow-A_{-(m+1)}^{+}$and $A_{m}^{+} \leftrightarrow-A_{-(m+1)}^{-}$. A second application reproduces the original operators; i.e., this transformation is an involution from the point of view of the two families of the Darboux transformations that we discussed. The ladder operators $\mathcal{C}_{m}^{ \pm}$ are invariant under this transformation. On the other hand, the stationary Schrödinger equation for the isotonic oscillator $L_{m}^{\text {iso }} \psi(x)=E \psi(x)$ is invariant under the transformation $x \rightarrow i x$ accompanied by a change $E \rightarrow-E$. Under this transformation $A_{m}^{ \pm} \rightarrow-i A_{-m}^{ \pm}, A_{-m}^{ \pm} \rightarrow-i A_{m}^{ \pm}$, and $\mathcal{C}_{m}^{-} \rightarrow-\mathcal{C}_{m}^{+}$, $\mathcal{C}_{m}^{+} \rightarrow-\mathcal{C}_{m}^{-}$. This transformation of the ladder operators reflects the fact that the invariance of the stationary Schrödinger equation for an isotonic oscillator also requires the indicated change of the energy sign. The double application of this transformation to the intertwining operators provokes just their multiplication by the phase -1 ; i.e., it also is the involution.

\section{DUAL SCHEMES AND MIRROR DIAGRAMS}

Here, we describe the construction of dual schemes of DC transformations that can conveniently be presented by a mirror diagram. Via a "charge conjugation" procedure, the mirror diagram allows us, in turn, to easily reconstruct a dual partner if one of the two schemes is given. The dual schemes and mirror diagrams will play a key role in the constructions of the next two sections.
In the previous section we saw that the isotonic oscillator systems can be generated from the half-harmonic oscillator $L_{0}^{\text {iso }}=L_{0}$ by applying to it DC transformations that use as the sets of seed states either only physical eigenstates of $L_{0}$, or only its specific nonphysical eigenstates that are obtained from the physical states by the spatial Wick rotation. We shall show now that these two schemes can be related one to another via a chain of intermediate DC schemes that are based on mixed sets of the physical and nonphysical eigenstates. This will be done by us for the pairs of DC transformations of a more general form which will be used in subsequent sections for construction of the rational extensions of the isotonic oscillator systems. To this aim we first note that the sets indicated above of physical and nonphysical eigenstates of the half-harmonic oscillator $L_{0}$ are also the eigenstates of the original quantum harmonic oscillator $L^{\text {osc }}$. Then, let us consider the DC transformation generated by a set of nonphysical eigenstates $\left(-n_{m}, \ldots,-n_{1}\right)$ of $L^{\text {osc }}$. We assume here that $n_{m}>\cdots>n_{1}>0$, and we do not preoccupy about zeros of the corresponding Wronskian $W\left(-n_{m}, \ldots,-n_{1}\right)$. It is rather natural to call such a scheme "negative." The scheme that will involve in the set of seed states $\left(n_{1}^{\prime}, \ldots, n_{l}^{\prime}\right)$, $0<n_{1}^{\prime}<\cdots<n_{l}^{\prime}$, only physical eigenstates of $L^{\text {osc }}$ we call "positive." Using the properties of the Wronskians described in Appendix A, we can write the relation $W\left(-n_{m}, \ldots,-n_{1}\right)=W\left(-0, \widetilde{-0},-n_{m}, \ldots,-n_{1}\right)$. As before, an equality between the corresponding Wronskians implies here the equality modulo a nonzero constant multiplier that has no effect on the corresponding DC transformations. Since the state $(-0)=\psi_{0}^{-}$generates, according to (A1), the first order differential operator $-a^{+}$, and $a^{+} \widetilde{\psi_{0}^{-}}=\psi_{0}$ [38], we obtain for $W\left(-n_{m}, \ldots,-n_{1}\right)$ the equivalent representations

$$
\begin{aligned}
e^{\frac{x^{2}}{2}} & W\left(a^{+}(\widetilde{-0}), a^{+}\left(-n_{m}\right), \ldots, a^{+}\left(-n_{1}\right)\right) \\
& =e^{\frac{x^{2}}{2}} W\left(0,-\left(n_{m}-1\right), \ldots,-\left(n_{1}-1\right)\right) .
\end{aligned}
$$

Having in mind that the DC transformation generates an additional potential term $-2(\ln W)^{\prime \prime}$, we see that the negative scheme $\left(-n_{m}, \ldots,-n_{1}\right)$ and the scheme $\left(0,-\left(n_{m}-1\right), \ldots,-\left(n_{1}-1\right)\right)$, which involves a mixed set of physical and nonphysical eigenstates of $L^{\text {osc }}$, generate the same quantum Hamiltonian up to a constant relative shift equal to 2 . Let us assume that $\left(n_{1}-1\right)>0$. In this case the last argument of the Wronskian in the equality in (3.1) will be a nonphysical eigenstate of $L^{\text {osc }}$ different from $(-0)$. Then we have a relation

$$
\begin{aligned}
& W\left(0,-\left(n_{m}-1\right), \ldots,-\left(n_{1}-1\right)\right) \\
& \quad=W\left(-0, \widetilde{-0}, 0,-\left(n_{m}-1\right), \ldots,-\left(n_{1}-1\right)\right) .
\end{aligned}
$$

The chain of relations of the form (3.1) can be iterated, and we obtain 
$W\left(-n_{m}, \ldots,-n_{1}\right)=e^{x^{2}} W\left(0,1,-\left(n_{m}-2\right), \ldots,-\left(n_{1}-2\right)\right)$.

$W(-2,-3,-4,-5,-8,-9,-11)=e^{6 x^{2}} W(1,4,5,10,11)$

In this second step the power of the exponent is multiplied by two, and now we have some new mixed scheme that includes two physical eigenstates of $L^{\text {osc }}$. If $n_{1}-2>0$, we repeat again the procedure till the moment when in the last argument of the Wronskian we obtain the nonphysical eigenstate $(-0)$. When this happens, we move this state to the first position in the Wronskian, and we use the relation $W\left(-0,0, \ldots,-\left(n_{1}-k\right)\right)=e^{x^{2} / 2} W\left(1, \ldots,-\left(n_{1}-(k+1)\right)\right)$. No matter whether the last argument in the Wronskian is a nonphysical state different from $(-0)$ or equal to it, we apply here one of the two corresponding algorithms described above and stop the chain of the transformations and equalities when all the arguments of the Wronskian will be physical eigenstates of the harmonic oscillator. In such a way we arrive finally at the relation of the form

$$
W(\underbrace{-n_{m}, \ldots,-n_{1}}_{n_{-}})=e^{\left(n_{m}+1\right) x^{2} / 2} W(\underbrace{n_{1}^{\prime}, \ldots, n_{m}^{\prime}=n_{m}}_{n_{+}}),
$$

where $0<n_{1}^{\prime}<\cdots<n_{m}^{\prime}=n_{m}$. This relation means that the negative scheme $\left(-n_{m}, \ldots,-n_{1}\right)$ with $n_{-}$seed states is dual to the positive scheme $\left(n_{1}^{\prime}, \ldots, n_{m}^{\prime}=n_{m}\right)$ with $n_{+}=$ $n_{m}+1-n_{-}$seed states, and that the corresponding DC transformations generate the same Hamiltonian operator modulo a relative constant shift $2\left(n_{m}+1\right)$.

In the simplest case we obtain in the described way the chain of relations

$$
\begin{aligned}
(-1) & =W(-0, \widetilde{-0},-1)=e^{x^{2} / 2} W\left(a^{+}(\widetilde{-0}), a^{+}(-1)\right) \\
& =e^{x^{2} / 2} W(0,-0)=e^{x^{2} / 2} W(-0,0) \\
& =e^{x^{2}} W\left(a^{+}(0)\right)=e^{x^{2}} \cdot(1) .
\end{aligned}
$$

This is generalized to a relation

$$
\begin{aligned}
& W\left(-1,-3, \ldots,-\left(2 n_{m}-1\right),-\left(2 n_{m}+1\right)\right) \\
& \quad=e^{\left(n_{m}+1\right) x^{2}} W\left(1,3, \ldots, 2 n_{m}-1,2 n_{m}+1\right),
\end{aligned}
$$

which corresponds to the pair of relations (2.4) and (2.14). Here we also used the property that the permutation of arguments in the Wronskians has no effect for the corresponding DC transformation. Another useful relation is $(-n)=W(-n)=e^{(n+1) x^{2} / 2} W(1,2, \ldots, n)$. This relation and the corresponding duality of the schemes $(-n)$ and $(1,2, \ldots, n)$, which we indicate by $(-n) \sim(1,2, \ldots, n)$, corresponds to isospectral deformation of the isotonic oscillator $L_{1}^{\text {iso }}$ and will be discussed in the next section. The particular relation and the duality of the corresponding schemes $(-2,-3,-4,-5,-8,-9,-11) \sim(1,4,5,10,11)$ will be used by us in an example of Sec. V.

We could start, instead, from some positive scheme. Then we introduce into the set of arguments of the Wronskian the pair of the states 0 and $\tilde{0}$, and use the relations $A_{0}^{-}=a^{-}, a^{-}(\tilde{0})=(-0), a^{-}(n)=(n-1)$, $W(0, n, \ldots)=e^{-x^{2} / 2} W\left(a^{-}(n), \ldots\right)$. The final result will be presented by a relation of the same form as (3.4).

The described procedure of construction of the dual schemes can be summarized and presented in the form of the corresponding mirror diagrams. Particularly, the duality of the schemes corresponding to relation (3.7) is presented by the mirror diagram shown in Fig. 1. The mirror diagram and the corresponding dual schemes are constructed in the following way. Let us take a positive scheme given by the set of the seed states $\left(n_{1}, \ldots, n_{m}\right)$ with $0<n_{1}<\cdots<n_{m}$. This scheme we present by the upper horizontal line, where we mark by unfilled, white circles the ground state $(0)$ of $L^{\text {osc }}$ and all physical eigenstates with a positive index less than $n_{m}$, which do not belong to the set of numbers $n_{1}, \ldots, n_{m}$. The states $n_{1}, \ldots, n_{m}$, which are selected as the seed states, we mark by filled, black circles. The dual, negative scheme is presented by the lower horizontal line, where the nonphysical states $(-n)$ which are used or not used in the scheme as the seed states are marked similarly by black and white circles, respectively. The circles in the negative scheme are obtained from those in the positive scheme by a kind of a charge conjugation procedure: below a white (black) circle in the upper line we put a black (white) circle in the lower line. Then we assign the set of consecutive numbers $\left(-n_{m},-n_{m}+1, \ldots,-1,-0\right)$ to the circles in the lower line by putting $\left(-n_{m}\right)$ below the state (0) in the upper line and $(-0)$ below the state $n_{m}$. If, instead, the negative scheme is given, we present it by the lower line and reconstruct the upper line of the dual, positive scheme in the obvious way by inverting the described procedure of the charge conjugation.

Let us denote the number of black circles in the upper line by $n_{+}$. Then the number of black circles in the lower line will be $n_{-}=n_{m}+1-n_{+}$. Remembering that each physical eigenstate $(n)$ carries the exponential factor $e^{-x^{2} / 2}$,

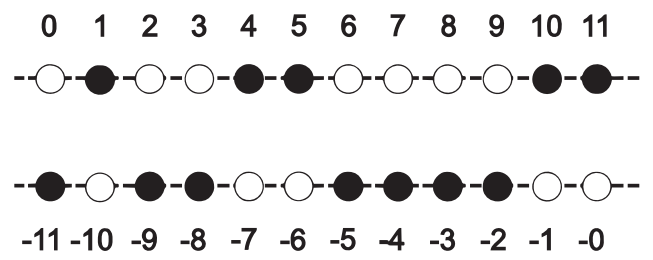

FIG. 1. The mirror diagram for the dual schemes in (3.7). 
while nonphysical state $(-n)$ carries the factor $e^{x^{2} / 2}$, we obtain the relation between Wronskians of the dual schemes in the form $W\left(-n_{m}, \ldots,-n_{1}\right) e^{-\left(n_{m}+1-n_{+}\right) x^{2} / 2}=$ $W\left(n_{1}^{\prime}, \ldots, n_{m}\right) e^{n_{+} x^{2} / 2}$ that corresponds to (3.4).

In order for dual schemes to produce the potentials that will describe nonsingular rational deformations of the conformal mechanics systems, the seed states have to be chosen in such a way that the corresponding Wronskians will have no zeros on the half-line $(0, \infty)$. In the next two sections, we describe how nonsingular dual schemes can be designed, and use them for the construction of ladder operators for corresponding rationally deformed isotonic oscillator systems.

\section{COMPLETELY ISOSPECTRAL RATIONAL DEFORMATIONS}

In Sec. II, we noticed that the selection of a set of $m$ nonphysical eigenstates $\psi_{2 l+1}^{-}, l=0,1, \ldots, m-1$, of the half-oscillator $L_{0}^{\text {osc }}$ as the seed states for the DC transformation produces the isotonic oscillator system $L_{m}^{\text {iso }}$. In this section we show that the choice of the same set of nonphysical eigenstates as the seed states but taken with gaps in the set of values of the parameter $l$ generates completely isospectral deformations of the isotonic oscillator systems. In spite of the complete isospectrality with the corresponding isotonic oscillator of index $m$, each such system will be characterized by peculiar lowering and raising ladder operators that will be spectrum-generating operators of differential order higher than two. For each completely isospectral deformation of conformal mechanics system we also construct an additional pair of ladder operators whose analogs will play an important role later for nonisospectral deformations of the isotonic oscillator systems.

The generation of completely isospectral rational deformations of the isotonic oscillator system $L_{m}^{\text {iso }}$ is based on the relation

$$
\begin{aligned}
& W\left(\psi_{2 l_{1}+1}^{-}, \psi_{2 l_{2}+1}^{-}, \ldots, \psi_{2 l_{m}+1}^{-}\right) \\
& \quad=e^{m x^{2} / 2} x^{m(m+1) / 2} f_{\left(-\left(2 l_{1}+1\right), \ldots,-\left(2 l_{m}+1\right)\right)}(x),
\end{aligned}
$$

where $f_{\left(-\left(2 l_{1}+1\right), \ldots,-\left(2 l_{m}+1\right)\right)}(x)$ is an even polynomial function with no real roots, and $0 \leq l_{1}<l_{2}<\cdots<l_{m}$; see Appendix C. In the case of the choice of the nonphysical eigenstates with indices given by a consecutive set $l_{1}=0, l_{2}=1, \ldots, l_{m}=m-1$ without any gap, polynomial function $f$ is reduced just to a nonzero constant, and Wronskian (4.1) generates by means of relation (2.14) the isotonic oscillator system $L_{m}^{\text {iso }}$ shifted for a constant $-2 m$. The appearance of any gap in the set of values $0 \leq l_{1}<$ $l_{2}<\cdots<l_{m}$ gives rise to a nontrivial polynomial $f(x)$ of even order that generates a completely isospectral deformation of the shifted isotonic oscillator system $L_{m}^{\text {iso }}-2 m$.
The corresponding Schrödinger operator generated by the DC transformation has the form

$$
\begin{aligned}
L_{\left(-\left(2 l_{1}+1\right), \ldots,-\left(2 l_{m}+1\right)\right)}= & L_{m}^{\text {iso }}-2\left(\ln f_{\left(-\left(2 l_{1}+1\right), \ldots,-\left(2 l_{m}+1\right)\right)}\right)^{\prime \prime} \\
& -2 m,
\end{aligned}
$$

and describes the isotonic Hamiltonian extended with a nonsingular on the half-line $x>0$ rational term. The nonsingular character of the additional rational term is related to the nature of the polynomial function $f_{\left(-\left(2 l_{1}+1\right), \ldots,-\left(2 l_{m}+1\right)\right)}(x)$ described above. In (4.2), the lower index in the notation of the Hamiltonian operator indicates what (nonphysical in this case) eigenstates of the quantum harmonic oscillator are used as the seed states in the corresponding DC transformation. A similar notation will be used below for the intertwining operators being generators of the corresponding Dabroux-Crum transformations. The complete isospectrality of (4.2) to the shifted isotonic oscillator $L_{m}^{\text {iso }}$ takes place because no new normalizable states are aggregated in this new system and its spectrum, up to a global shift, has to coincide with the spectrum of $L_{0}$.

For each fixed integer $m \geq 1$ we have an infinite number of possibilities to choose $m$ seed states in the form of the set of nonphysical eigenstates of $L^{\text {osc }}$. In this way we can construct an infinite family of the deformed isotonic oscillator systems that will be completely isospectral to the shifted isotonic oscillator system $L_{m}^{\text {iso }}-2 m$. For each such system, there is another dual DC transformation to generate it. The existence of the dual DC schemes is essential for the construction of the ladder operators for the rationally extended isotonic oscillator systems.

As an example, consider the case of the simplest deformed isotonic oscillator system generated via the Darboux transformation based on the nonphysical eigenstate $\psi_{3}^{-}=\left(2 x^{3}+3 x\right) e^{x^{2} / 2}$ of the half-harmonic oscillator $L_{0}^{\text {iso }}$. In this case $f_{(-3)}(x)=2 x^{2}+3$, and Eq. (4.2) reduces to

$$
L_{(-3)}=L_{1}^{\text {iso }}-2+8 \frac{2 x^{2}-3}{\left(2 x^{2}+3\right)^{2}} .
$$

By the method of the mirror diagram, we find that up to a constant shift, the system can be generated alternatively by the DC transformation based on the set $\left\{\psi_{1}, \psi_{2}, \psi_{3}\right\}$ of physical eigenstates of the quantum harmonic oscillator, $L_{(1,2,3)}=L_{(-3)}+8$. The states $\psi_{1}$ and $\psi_{3}$ here are the two lowest physical eigenstates of the half-harmonic oscillator, whereas the state $\psi_{2}$ is a nonphysical eigenstate of $L_{0}=$ $L_{0}^{\text {iso }}$ that does not satisfy the boundary condition at $x=0$.

Though the schemes based on the two different sets of seed states $(-3)$ and $(1,2,3)$ generate, up to a constant shift, the same system, the corresponding associated intertwining operators are essentially different. The intertwiners 


$$
\begin{aligned}
& A_{(-3)}^{-}=\psi_{3}^{-} \frac{d}{d x} \frac{1}{\psi_{3}^{-}}=A_{-1}^{-}-\frac{4 x}{2 x^{2}+3}, \\
& A_{(-3)}^{+}=-\frac{1}{\psi_{3}^{-}} \frac{d}{d x} \psi_{3}^{-}=A_{-1}^{+}-\frac{4 x}{2 x^{2}+3},
\end{aligned}
$$

are the extensions of the first order differential operators $A_{-1}^{ \pm}$ of the form (2.15) with $m=1$. The appearance of the operators $A_{-1}^{ \pm}$in the structure of the DC generators $A_{(-3)}^{ \pm}$in (4.4) reflects the fact that the system (4.3) represents a rational extension of the isotonic oscillator system $L_{1}^{\text {iso }}$. The additional rational term $-4 x /\left(2 x^{2}+3\right)$ in (4.4) is regular on all the half-axis $x>0$ and tends to zero for $x \rightarrow 0$ and $x \rightarrow+\infty$. We have the factorization relations $A_{(-3)}^{+} A_{(-3)}^{-}=L_{0}+7, \quad A_{(-3)}^{-} A_{(-3)}^{+}=L_{(-3)}+7=L_{(1,2,3)}-1$. In correspondence with them, $A_{(-3)}^{-}$intertwines the Hamiltonian operators $L_{0}$ and $L_{(-3)}$,

$A_{(-3)}^{-} L_{0}=L_{(-3)} A_{(-3)}^{-}=\left(L_{(1,2,3)}-2 \Delta E^{\text {iso }}\right) A_{(-3)}^{-}$,

where the constant $2 \Delta E^{\text {iso }}=8$ will reveal itself in the properties of the ladder operators. The intertwining relation for $A_{(-3)}^{+}$is obtained by Hermitian conjugation.

The systems $L_{0}$ and $L_{(1,2,3)}$ are also intertwined by the third order operators $\mathbb{A}_{(1,2,3)}^{-}$and $\mathbb{A}_{(1,2,3)}^{+}=\left(\mathbb{A}_{(1,2,3)}^{-}\right)^{\dagger}$, where the operator $\mathbb{A}_{(1,2,3)}^{-}$is uniquely specified by its kernel: $\operatorname{ker} \mathbb{A}_{(1,2,3)}^{-}=\operatorname{span}\left\{\psi_{1}, \psi_{2}, \psi_{3}\right\}$. Notice that the factorized form of the operators $\mathbb{A}_{(1,2,3)}^{ \pm}$is not unique, but it is not important for us at the moment. In such notations, the operators $\mathbb{A}_{m}^{-}$and $\mathbb{A}_{-m}^{-}$, which we discussed before for generation of isotonic oscillator systems, take, in correspondence with Eqs. (2.4) and (2.14), the form $\mathbb{A}_{m}^{-}=\mathbb{A}_{(1,3, \ldots, 2 m-1)}^{-}$and $\mathbb{A}_{-m}^{-}=\mathbb{A}_{(-1,-3, \ldots,-(2 m-1))}^{-}$. We have the intertwining relation $\mathbb{A}_{(1,2,3)}^{-} L_{0}=L_{(1,2,3)} \mathbb{A}_{(1,2,3)}^{-}=$ $\left(L_{(-3)}+8\right) \mathbb{A}_{(1,2,3)}^{-}$, and the conjugate relation for $\mathbb{A}_{(1,2,3)}^{+}$.

Analogous to the case of the isotonic oscillator systems, we can construct the ladder operators for the system $L_{(-3)}=L_{(1,2,3)}-8$ by Darboux dressing of the ladder operators of the half-harmonic oscillator. Doing this with the first order intertwining operators, we obtain

$$
\mathcal{A}^{ \pm}=A_{(-3)}^{-}\left(a^{ \pm}\right)^{2} A_{(-3)}^{+} \text {. }
$$

These operators together with the Hamiltonian $L_{(-3)}$ generate a nonlinear deformation of the conformal symmetry given by the commutation relations

$$
\begin{aligned}
{\left[L_{(-3)}, \mathcal{A}^{ \pm}\right] } & = \pm 4 \mathcal{A}^{ \pm} \\
{\left[\mathcal{A}^{-}, \mathcal{A}^{+}\right] } & =16\left(L_{(-3)}+3\right)\left(L_{(-3)}+7\right)\left(L_{(-3)}+1 / 2\right) .
\end{aligned}
$$

The roots of the fourth order polynomial in the relation

$$
\mathcal{A}^{+} \mathcal{A}^{-}=\left(L_{(-3)}+7\right)\left(L_{(-3)}+3\right)\left(L_{(-3)}-1\right)\left(L_{(-3)}-3\right)
$$

correspond to eigenstates of $L_{(-3)}$ that belong to the kernel of the lowering operator,

$\operatorname{ker} \mathcal{A}^{-}=\operatorname{span}\left\{A_{(-3)}^{-} \widetilde{\psi_{3}^{-}}, A_{(-3)}^{-} \psi_{1}^{-}, A_{(-3)}^{-} \psi_{0}, A_{(-3)}^{-} \psi_{1}\right\}$.

The last state $A_{(-3)}^{-} \psi_{1}=\mathbb{A}_{(1,2,3)}^{-} \psi_{5}$ describes here the ground state of $L_{(-3)}$ of eigenvalue $E=3$. In comparison with the second order ladder operator $\left(a^{-}\right)^{2}$ of the halfharmonic oscillator, the fourth order lowering operator $\mathcal{A}^{-}$ contains besides the ground state three nonphysical eigenstates of the Hamiltonian $L_{(-3)}$ instead of one. The roots in the alternative product

$\mathcal{A}^{-} \mathcal{A}^{+}=\left(L_{(-3)}+11\right)\left(L_{(-3)}+7\right)\left(L_{(-3)}+3\right)\left(L_{(-3)}+1\right)$

correspond to eigenvalues of the eigenstates of $L_{(-3)}$ which appear in the kernel of the raising ladder operator,

$\operatorname{ker} \mathcal{A}^{+}=\operatorname{span}\left\{A_{(-3)}^{-} \psi_{5}^{-}, A_{(-3)}^{-} \widetilde{\psi_{3}^{-}}, A_{(-3)}^{-} \psi_{1}^{-}, A_{(-3)}^{-} \psi_{0}^{-}\right\}$.

All the states in this kernel are nonphysical. In correspondence with the described properties of the ladder operators (4.6) they are the spectrum-generating operators for the system $L_{(-3)}$ : acting by them on any physical eigenstate of $L_{(-3)}$, we can generate any other physical eigenstate. The kernels of the ladder operators contain here the same nonphysical eigenstate $A_{(-3)}^{-} \widetilde{\psi_{3}^{-}}=A_{(-3)}^{-} \psi_{1}^{-}$. Below we shall see that in the case of nonisospectral rational deformations of the isotonic oscillator systems the kernels of analogs of such lowering and raising ladder operators contain some common physical eigenstates.

In a similar way, one can construct the ladder operators for $L_{(-3)}$ via Darboux dressing of the ladder operators $\left(a^{ \pm}\right)^{2}$ of $L_{0}$ by the third order intertwining operators, $\mathcal{B}^{ \pm}=$ $\mathbb{A}_{(1,2,3)}^{-}\left(a^{ \pm}\right)^{2} \mathbb{A}_{(1,2,3)}^{+},\left[L_{(-3)}, \mathcal{B}^{ \pm}\right]= \pm 4 \mathcal{B}^{ \pm}$. However, these differential operators of order eight are not independent and reduce to the fourth order ladder operators (4.6) multiplied by the second order polynomials in the Hamiltonian,

$$
\mathcal{B}^{-}=\mathcal{A}^{-}\left(L_{(-3)}+1\right)\left(L_{(-3)}+5\right) \quad \text { and } \quad \mathcal{B}^{+}=\left(\mathcal{B}^{-}\right)^{\dagger} .
$$

In correspondence with these relations, the operator $\mathcal{B}^{-}$ annihilates four nonphysical eigenstates $A_{(-3)}^{-} \psi_{0}^{-}, A_{(-3)}^{-} \widetilde{\psi_{0}^{-}}$, 
$A_{(-3)}^{-} \psi_{2}^{-}$, and $A_{(-3)}^{-} \widetilde{\psi_{2}^{-}}$of the quantum Hamiltonian $L_{(-3)}$ in addition to those states that constitute the kernel of the ladder operator $\mathcal{A}^{-}$. Analogously, the kernel of $\mathcal{B}^{+}$is composed from nonphysical states of the kernel of the operator $\mathcal{A}^{+}$extended by four nonphysical eigenstates $A_{(-3)}^{-} \psi_{4}^{-}, \quad A_{(-3)}^{-} \widetilde{\psi_{4}^{-}}, \quad A_{(-3)}^{-} \psi_{2}^{-}, \quad$ and $A_{(-3)}^{-} \widetilde{\psi_{2}^{-}}$of the Hamiltonian $L_{(-3)}$. Since all the additional states from the kernels of the operators $\mathcal{B}^{-}$and $\mathcal{B}^{+}$in comparison with the kernels of the ladder operators $\mathcal{A}^{-}$and $\mathcal{A}^{+}$are nonphysical, the $\mathcal{B}^{-}$and $\mathcal{B}^{+}$are also the spectrum-generating operators of the system $L_{(-3)}$. So, in this case one can conclude that the order eight ladder operators $\mathcal{B}^{ \pm}$are, in fact, equivalent to the fourth order ladder operators (4.6) when they act on physical eigenstates. We shall see later that in the case of the nonisospectral rational extensions of the isotonic oscillator systems different Darboux-dressing procedures produce nonequivalent pairs of the ladder operators which will reflect different properties of the corresponding quantum systems.

Consider the construction of yet another pair of ladder operators for the same system $L_{(-3)}$ by Darboux dressing the ladder operators of the isotonic oscillator $L_{2}^{\text {iso }}$. For this we first note that the iterative nature of the DC transformation allows us to present the third order intertwining operators $\mathbb{A}_{(1,2,3)}^{ \pm}$in the following particular factorized form:

$\mathbb{A}_{(1,2,3)}^{-}=A_{(2)}^{(1,3)-} \mathbb{A}_{(1,3)}^{-}, \quad \mathbb{A}_{(1,2,3)}^{+}=\mathbb{A}_{(1,3)}^{+} A_{(2)}^{(1,3)+}$.

Here the second order differential operators $\mathbb{A}_{(1,3)}^{-}=\mathbb{A}_{2}^{-}=$ $A_{2}^{-} A_{1}^{-}$and $\mathbb{A}_{(1,3)}^{+}=\left(\mathbb{A}_{(1,3)}^{-}\right)^{\dagger}=\mathbb{A}_{2}^{+}$correspond exactly to the particular case $m=2$ of the operators (2.11) that intertwine the half-harmonic oscillator $L_{0}$ and the shifted isotonic oscillator system $L_{2}$. The first order differential operators $A_{(2)}^{(1,3)-}$ and $A_{(2)}^{(1,3)+}$ are given by relations $A_{(2)}^{(1,3)-}=$ $\left(\mathbb{A}_{2}^{-} \psi_{2}\right) \frac{d}{d x}\left(\mathbb{A}_{2}^{-} \psi_{2}\right)^{-1}, A_{(2)}^{(1,3)+}=\left(A_{(2)}^{(1,3)-}\right)^{\dagger}$. Representation (4.13) can be related to the Wronskian identity $W(1,2,3)=-W(1,3,2)$, and corresponds to a general rule of association of the $n$th order intertwining operators with the DC transformation of order $n$ described in Appendix A. The upper index in the operator $A_{(2)}^{(1,3)-}$ indicates the seed states of the harmonic oscillator we use for the construction of the second order operators $\mathbb{A}_{2}^{ \pm}$, and the lower index indicates the eigenstate $\psi_{2}$ of $L^{\text {osc }}$ to which we apply the operator $\mathbb{A}_{2}^{-}$to generate finally the operators $A_{(2)}^{(1,3)-}$ and $A_{(2)}^{(1,3)+}$.

As $\mathbb{A}_{2}^{-} \psi_{2}=x^{-2}\left(3+2 x^{2}\right) e^{-x^{2} / 2}$ is a (nonphysical) eigenstate of $L_{2}$, which can be obtained by applying the symmetry transformation $m \rightarrow-m-1$ to the first excited state of $L_{2}$ by means of Eq. (B2), the operators $A_{(2)}^{(1,3)-}$ and
$A_{(2)}^{(1,3)+}$ factorize the appropriately shifted Hamiltonian operators $L_{2}$ and $L_{(1,2,3)}$,

$A_{(2)}^{(1,3)+} A_{(2)}^{(1,3)-}=L_{2}-5, \quad A_{(2)}^{(1,3)-} A_{(2)}^{(1,3)+}=L_{(1,2,3)}-5$,

and intertwine them,

$A_{(2)}^{(1,3)-} L_{2}=L_{(1,2,3)} A_{(2)}^{(1,3)-}, \quad A_{(2)}^{(1,3)+} L_{(1,2,3)}=L_{2} A_{(2)}^{(1,3)+}$.

Based on these properties and relations, we can construct the ladder operators for the Hamiltonian operator $L_{(1,2,3)}=$ $L_{(-3)}+8$ by Darboux dressing the ladder operators $\mathcal{C}_{2}^{ \pm}$of the isotonic oscillator system $L_{2}, \tilde{\mathcal{B}}^{ \pm}=A_{(2)}^{(1,3)-} \mathcal{C}_{2}^{ \pm} A_{(2)}^{(1,3)+}$. A simple calculation of the products $\tilde{\mathcal{B}}^{-} \tilde{\mathcal{B}}^{+}$and $\tilde{\mathcal{B}}^{+} \tilde{\mathcal{B}}^{-}$shows that the kernels of the ladder operators $\tilde{\mathcal{B}}^{-}$and $\tilde{\mathcal{B}}^{+}$coincide with the kernels of the operators $\mathcal{A}^{-}$and $\mathcal{A}^{+}$, and we find that the operators $\tilde{\mathcal{B}}^{ \pm}$are the same ladder operators as (4.6): $\tilde{\mathcal{B}}^{ \pm}=-\mathcal{A}^{ \pm}$.

Intuitively, one can expect that for each system completely isospectral to the half-harmonic oscillator $L_{0}$, all the ladder operators constructed by Darboux dressing do the same work, and that they are related between themselves by a simple multiplication with a polynomial in the corresponding Hamiltonian. This regularity appears for both the isotonic oscillator systems and for their isospectral rational extensions. However, having in mind the analogy with the rationally extended quantum harmonic oscillator systems [45], we cannot expect the same picture in nonisospectral deformations of the isotonic oscillator systems having gaps in the spectra.

As the first and third order operators $A_{(-3)}^{ \pm}$and $\mathbb{A}_{(1,2,3)}^{ \pm}$ intertwine the half-harmonic oscillator with the system $L_{(-3)}$ with a nonzero relative shift, we can construct yet another pair of the ladder operators for the quantum system $L_{(-3)}$,

$$
\mathcal{C}^{-}=\mathbb{A}_{(1,2,3)}^{-} A_{(-3)}^{+}, \quad \mathcal{C}^{+}=A_{(-3)}^{-} \mathbb{A}_{(1,2,3)}^{+} .
$$

These fourth order differential operators have a structure similar to the factorized structure of the ladder operators $\mathcal{C}_{m}^{ \pm}$for isotonic oscillator systems that we considered in Sec. II. Unlike the ladder operators $\mathcal{A}^{ \pm}, \mathcal{B}^{ \pm}$, and $\tilde{\mathcal{B}}^{ \pm}$, the $\mathcal{C}^{ \pm}$satisfy the commutation relations $\left[L_{(-3)}, \mathcal{C}^{ \pm}\right]=$ $\pm 2 \Delta E^{\text {iso }} \mathcal{C}^{ \pm}$, where the coefficient $2 \Delta E^{\text {iso }}=8$ corresponds to the relative shift between the operators $L_{(1,2,3)}$ and $L_{(-3)}$ and is equal to the double distance between the energy levels in the spectrum of the rationally extended isotonic system $L_{(-3)}$. They generate the following polynomial deformation of the conformal symmetry: 


$$
\begin{aligned}
{\left[L_{(-3)}, \mathcal{C}^{ \pm}\right] } & = \pm 8 \mathcal{C}^{ \pm} \\
{\left[\mathcal{C}^{-}, \mathcal{C}^{+}\right] } & =32\left(L_{(-3)}^{3}+6 L_{(-3)}^{2}-L_{(-3)}+30\right) .
\end{aligned}
$$

The kernel of the lowering ladder operator is

$\operatorname{ker} \mathcal{C}^{-}=\operatorname{span}\left\{\left(\psi_{(-3)}^{-}\right)^{-1}, A_{(-3)}^{-} \psi_{1}, A_{(-3)}^{-} \psi_{2}, A_{(-3)}^{-} \psi_{3}\right\}$.

Here $A_{(-3)}^{-} \psi_{1}=\mathbb{A}_{(1,2,3)}^{-} \psi_{5}$ and $A_{(-3)}^{-} \psi_{3}=\mathbb{A}_{(1,2,3)}^{-} \psi_{7}$ are the ground and the first exited states of $L_{(-3)}$. All the states in the kernel of the raising ladder operator are nonphysical:

$\operatorname{ker} \mathcal{C}^{+}=\operatorname{span}\left\{A_{(-3)}^{-} \psi_{7}^{-}, A_{(-3)}^{-} \psi_{2}^{-}, A_{(-3)}^{-} \psi_{1}^{-}, A_{(-3)}^{-} \psi_{0}^{-}\right\}$.

As a result, the space of states of $L_{(-3)}$ is separated into two subspaces, on each of which the ladder operators $\mathcal{C}^{+}$ and $\mathcal{C}^{-}$act irreducibly. One subspace is spanned by the ground state and the corresponding excited eigenstates, $A_{(-3)}^{-} \psi_{4 l+1}=\left(\mathcal{C}^{+}\right)^{l}\left(A_{(-3)}^{-} \psi_{1}\right)=\left(\mathcal{A}^{+}\right)^{2 l}\left(A_{(-3)}^{-} \psi_{1}\right), \quad l=0$, $1, \ldots$. Another subspace corresponds to the first excited state and the infinite tower of the states produced from it by application of the raising operator $\mathcal{C}^{+}, A_{(-3)}^{-} \psi_{4 l+3}=$ $\left(\mathcal{C}^{+}\right)^{l}\left(A_{(-3)}^{-} \psi_{3}\right)=\left(\mathcal{A}^{+}\right)^{2 l}\left(A_{(-3)}^{-} \psi_{3}\right), l=0,1, \ldots$. Here, as in other relations, we imply equalities modulo nonzero multiplicative factors. The ladder operators $\mathcal{C}^{ \pm}$, unlike $\mathcal{A}^{ \pm}$, are therefore not spectrum-generating operators for the system $L_{(-3)}$. Notice that from the point of view of the basic properties of the ladder operators $\mathcal{C}^{ \pm}$, they are similar to the operators $\left(\mathcal{C}_{0}^{ \pm}\right)^{2}=\left(a^{ \pm}\right)^{4}$ in the case of the halfharmonic oscillator $L_{0}$, or to the operators $\tilde{\mathcal{C}}_{2}^{ \pm}$for isotonic oscillator $L_{2}^{\text {iso }}$. The essential difference here, however, is that the ladder operators $\mathcal{C}^{ \pm}$are independent from the spectrum-generating ladder operators $\mathcal{A}^{ \pm}$and have the same differential order equal to four. We shall see that for nonisospectral rational extensions of the isotonic oscillator systems the direct analogs of the operators $\mathcal{C}^{ \pm}$will constitute an inseparable part of the set of the spectrumgenerating operators.

Let us also note here yet another possibility to generate the same system $L_{(-3)}$. We first can choose the neighbor excited eigenstates $\psi_{2}$ and $\psi_{3}$ of the quantum harmonic oscillator as the seed states for the DC transformation. In this way we generate the following rational extension of the quantum harmonic oscillator:

$$
L_{(2,3)}=-\frac{d^{2}}{d x^{2}}+x^{2}-\frac{16 x^{2}\left(4 x^{4}-9\right)}{\left(3+4 x^{4}\right)^{2}}+4 .
$$

This system is described by the potential that is regular on all the real line. Its spectrum, up to the shift equal to four, is the same as of the harmonic oscillator $L^{\text {osc }}$ except for two missing energy levels between $E_{1}=7$ and $E_{4}=13$. We can modify then the system $L_{(2,3)}$ similar to the construction of the half-harmonic oscillator by introducing the infinite potential barrier at $x=0$ and restricting the domain for the semi-infinite interval $(0,+\infty)$. This is equivalent to removing all the even eigenstates and corresponding eigenvalues from the spectrum of the Hamiltonian operator $L_{(2,3)}$. We denote this intermediate system by $L_{(2,3)}^{1 / 2}$. This system has the equidistant spectrum with the double distance between energy levels in comparison with the spectrum of the quantum harmonic oscillator, and its ground state of energy $E_{1}=7$ is described by the wave function of the form $\psi=W(2,3,1) / W(2,3)$. Using this ground state of the obtained system $L_{(2,3)}^{1 / 2}$ as the seed state, we apply Darboux transformation to it. The partner system for $L_{(2,3)}^{1 / 2}$ that we produce in this way is exactly the system $L_{(1,2,3)}$.

The generation of the system $L_{(123)}$ can also be reinterpreted by taking $L_{1}$ as a starting point. For this we select the state $A_{1}^{-} \psi_{2}=\frac{1+2 x^{2}}{x} e^{-x^{2} / 2}$, singular at $x=0$, and the ground state $A_{1}^{-} \psi_{3}$. By virtue of iteration properties of the DC transformations, one can check that with the set of the seed states $\left\{A_{1}^{-} \psi_{2}, A_{1}^{-} \psi_{3}\right\}$ we produce the system $L_{(1,2,3)}$. The singular state $A_{1}^{-} \psi_{2}$ can be obtained by the same trick we already used by applying the transformation $m \rightarrow-(m+$ 1 ) to the first excited state of $L_{1}$. In what follows for us will also be important for the versions of such states obtained by additional transformation $x \rightarrow i x, E \rightarrow-E$.

It is worthwhile to note here that we constructed the fourth order ladder operators $\tilde{\mathcal{B}}^{ \pm}$by Darboux dressing the ladder operators of the isotonic oscillator $L_{2}$ though the system $L_{(-3)}$ is the isospectral rational deformation of the isotonic oscillator $L_{1}$. One can construct the pair of the ladder operators by Darboux dressing the ladder operators of the isotonic oscillator $L_{1}$, but it turns out to be related to the fourth order operators $\tilde{\mathcal{B}}^{ \pm}$in a simple way. The analogous phenomena of relation between different ladder operators obtainable via the DC dressing procedure happen also in more complicated cases of rational deformations of the isotonic oscillator systems. By this reason, let us also consider briefly the construction of yet another indicated pair of ladder operators. For this we first write down another factorization of the third order intertwining operator $\mathbb{A}_{(1,2,3)}^{-}: \quad \mathbb{A}_{(1,2,3)}^{-}=\mathbb{A}_{(2,3)}^{(1)-} A_{(1)}^{-} ;$cf. (4.13). Here $A_{(1)}^{-}=A_{1}^{-}$is the first order differential operator that intertwines $L_{0}$ and $L_{1}$, and $\mathbb{A}_{(2,3)}^{(1)-}$ is the second order differential operator that intertwines the systems $L_{1}$ and $L_{(1,2,3)}$. We can construct now the order six ladder operators for the system $L_{(-3)}=L_{(1,2,3)}-8$ by the DC dressing of the ladder operators of the isotonic oscillator $L_{1}: \hat{\mathcal{B}}^{ \pm}=\mathbb{A}_{(2,3)}^{(1)-} \mathcal{C}_{1}^{ \pm} \mathbb{A}_{(2,3)}^{(1)+}$. However, they are related to the fourth order ladder operators $\tilde{\mathcal{B}}^{ \pm}=-\mathcal{A}^{ \pm}$in a simple way: 
$\hat{\mathcal{B}}^{-}=\tilde{\mathcal{B}}^{-}\left(L_{(-3)}+1\right), \quad \hat{\mathcal{B}}^{+}=\tilde{\mathcal{B}}^{+}\left(L_{(-3)}+5\right)$.

The additional factors of the first order in Hamiltonian annihilate the corresponding nonphysical eigenstates of $L_{(-3)}$ of eigenvalues -1 and -5 . This means that the action of these six order ladder operators on physical states of the system $L_{(-3)}$ is, in fact, the same as of the ladder operators $\tilde{\mathcal{B}}^{ \pm}=-\mathcal{A}^{ \pm}$and $\mathcal{B}^{ \pm}$

Since the system $L_{(-3)}$ is completely isospectral to the isotonic oscillator $L_{1}$ having ladder operators of differential order two, there may appear a question of whether other ladder operators of differential order two do exist here. One can show, however, that the unique Hamiltonian operators admitting the existence of ladder operators of differential order two are those of the harmonic oscillator and the isotonic oscillator systems as well as their "anyonic" modifications of the form (2.3) with coefficient $m(m+1)$ changed there for $\nu(\nu+1)$ with $\nu>0$.

Consider briefly a more complicated case of the system $L_{(-3,-7)}$. This is an isospectral rational extension of $L_{2}^{\text {iso }}$ with potential given by Eq. (D1). The mirror diagram gives us an equivalent way to obtain the same, up to a shift, system by using the set of the seed states $(1,2,3,5,6,7)$ : $L_{(1,2,3,5,6,7)}=L_{(-3,-7)}+16$, where $16=4 \Delta E^{\text {iso }}$. The intertwining operators $\mathbb{A}_{(-3,-7)}^{-}$and $\mathbb{A}_{(1,2,3,5,6,7)}^{-}$satisfy the relations $\mathbb{A}_{(-3,-7)}^{-} L_{0}^{\text {iso }}=L_{(-3,-7)} \mathbb{A}_{(-3,-7)}^{-}$and $\mathbb{A}_{(1,2,3,5,6,7)}^{-} L_{0}^{\text {iso }}=$ $L_{(1,2,3,5,6,7)} \mathbb{A}_{(1,2,3,5,6,7)}$. Since here we also have two schemes, the procedure developed in the previous example is applicable for the construction of the ladder operators of the types $\mathcal{A}^{ \pm}, \mathcal{B}^{ \pm}$, and $\mathcal{C}^{ \pm}$. Besides, this system can be obtained by taking as a starting point the isotonic oscillator $L_{4}$ to which we apply the DC transformation based on the seed states $\mathbb{A}_{4}^{-} \psi_{2}$ and $\mathbb{A}_{4}^{-} \psi_{6}$, which are singular but have no zeros on the positive half-line. So, by dressing the operators $\mathcal{C}_{4}^{ \pm}$with the intertwining operators corresponding to this transformation, we also can assemble a pair of the ladder operators of the type $\tilde{\mathcal{B}}^{ \pm}$.

The described properties of the two particular examples of the rationally extended isotonic oscillator systems can be generalized and summarized as follows. No matter what set of the $m$ odd nonphysical eigenstates of the quantum harmonic oscillator $L^{\text {osc }}$ we select, the lower order ladder operators $\mathcal{A}^{ \pm}$obtained by Darboux dressing of the ladder operators of the half-harmonic oscillator are spectrum-generating operators for the rationally deformed isotonic oscillator system. They commute for a polynomial of order $2 m+1$ in the corresponding Hamiltonian operator with which they produce a deformation of the conformal $\mathfrak{g l}(2, \mathbb{R})$ symmetry of the type of $W$ algebra [51]. Other spectrum-generating ladder operators, which can be constructed on the basis of other DC schemes via the Darbox-dressing procedure, act on physical states in the same way as the operators $\mathcal{A}^{ \pm}$of order $2(m+1)$ and are equal to them modulo the multiplicative factor in the form of the polynomial in the Hamiltonian operator of the system. The ladder operators $\mathcal{C}^{ \pm}$constructed by "gluing" intertwining operators of the two dual schemes are not spectrum generating. Their properties are analogous to those of the ladder operators $\tilde{\mathcal{C}}_{m}^{ \pm}$in the isotonic oscillator $L_{m}^{\text {iso }}$ with $m>1$. Particularly, for the isospectral deformation of the system $L_{l_{m}+1}^{\text {iso }}$ based on the set of the seed states $\left(-\left(2 l_{1}+1\right),-\left(2 l_{2}+1\right), \ldots,-\left(2 l_{m}+1\right)\right)$ with $0 \leq l_{1}<$ $l_{2}<\cdots<l_{m}, l_{m} \geq 1$, the operator $\mathcal{C}^{-}$annihilates the lowest $l_{m}+1$ states in the spectrum of the system. The Hilbert space of the system $L_{\left(-\left(2 l_{1}+1\right), \ldots,-\left(2 l_{m}+1\right)\right)}$ is separated into $l_{m}+1$ subspaces in the form of the towers over these states which are invariant under the action of the ladder operators $\mathcal{C}^{ \pm}$. However, unlike $\tilde{\mathcal{C}}_{m}^{ \pm}$, here the ladder operators $\mathcal{C}^{ \pm}$are independent from the spectrum-generating operators $\mathcal{A}^{ \pm}$.

\section{GAPPED DEFORMATIONS}

To construct the isotonic oscillator systems and their isospectral rational deformations, we have introduced an infinite potential barrier into the quantum harmonic oscillator system at $x=0$. In this way we obtained the half-harmonic oscillator $L_{0}$, and then we applied to it a DC transformation generated on the basis of physical or certain nonphysical eigenstates of $L_{0}$ taken as the seed states. This procedure also allowed us to obtain different types of ladder operators for corresponding systems. In order to generate nonisospectral rational deformations and find their ladder operators, we repeat the procedure but by changing every time the half-harmonic oscillator $L_{0}$ for the half of the appropriately chosen rationally extended quantum harmonic oscillator system. The nonisospectrality corresponds here to the appearance of $N_{g}$ "valence bands," the highest of which is separated by an energy gap $n_{0} \Delta E^{\text {iso }}$ from the equidistant part of the spectrum with the spacing $\Delta E^{\text {iso }}=4$, where $n_{0} \geq 2$ is integer. In the case $N_{g} \geq 2$, a separated part of the spectrum will contain in addition $N_{g}-1$ energy gaps of dimensions $n_{i} \Delta E^{\text {iso }}, n_{i} \geq 2, i=1, \ldots, N_{g}-1$, which will separate $\left(N_{g}-1\right)$ groups of equidistant energy levels spaced by $\Delta E^{\text {iso }}=4$ inside each valence band with more than one state. By eliminating then not all but some number of the lowest consecutive energy levels from the valence bands by means of a DC transformation, as a result we obtain a certain deformation of the corresponding isotonic oscillator system. The general properties of the DC transformations will finally allow us to present the described two-step procedure in an equivalent form of a one-step DC transformation applied to the half-harmonic oscillator system.

The described general procedure is realized explicitly in the following way. Consider a quantum system 


$$
\begin{aligned}
L= & -\frac{d^{2}}{d x^{2}}+x^{2} \\
& -2\left(\ln W\left(l_{1}, l_{1}+1, l_{2}, l_{2}+1, \ldots, l_{m}, l_{m}+1\right)\right)^{\prime \prime}
\end{aligned}
$$

generated from the quantum harmonic oscillator by means of the DC transformation based on a set of the seed states $\left(l_{1}, l_{1}+1, l_{2}, l_{2}+1, \ldots, l_{m}, l_{m}+1\right)$ of $L^{\text {osc }}$, where we assume that $l_{1}>0$ and $l_{i}+1<l_{i+1}$. The quantum system defined by (5.1) has an even potential and describes a rationally extended quantum harmonic oscillator system. Its spectrum coincides with the spectrum of the quantum harmonic oscillator except the missing energy levels that correspond to the energies of the seed states; see Ref. [45] for the details. Then we introduce the potential barrier into the system (5.1) at $x=0$ by requiring that for $x>0$ the potential of the "halved" system $L^{1 / 2}$ coincides with the potential of (5.1) while for $x \leq 0, V(x)=+\infty$. This is equivalent to demanding that the wave functions of the physical states of the system $L^{1 / 2}$ disappear for $x \leq 0$. As a result, the eigenstates of the system $L^{1 / 2}$ on the half-line $x>0$ will be described by the odd eigenfunctions of the system (5.1). The spectrum of the system $L^{1 / 2}$ will be given by the numbers $E_{2 n+1}=4 n+3$, where $n$ takes all the values $n=0,1, \ldots$, except those values for which $2 n+1$ coincides with any of the odd integers contained in the set of the numbers that correspond to the indices $\left(l_{1}, l_{1}+1\right.$, $\left.l_{2}, l_{2}+1, \ldots, l_{m}, l_{m}+1\right)$ of the seed states used in the DC transformation for the system (5.1). This means that each state with an odd index that appears in the Wronskian in (5.1) removes an energy level in the spectrum of $L^{1 / 2}$. As a result, by controlling the choice of the set of numbers $l_{i}$, we can produce a halved rationally extended quantum harmonic oscillator system with gaps of arbitrary number and size, at will. The even eigenstates are implemented in the corresponding DC transformation effectively to avoid singularities in the Hamiltonian operator (5.1). After that, we realize an additional DC transformation based on a set of consecutive lowest eigenstates of the system $L^{1 / 2}$ starting from its ground state. These states in the set eliminate the corresponding lower lying energy levels in the spectrum of the partner system obtained from the system $L^{1 / 2}$. If in such a way we eliminate all the energy levels from the separated part of the spectrum of $L^{1 / 2}$, depending on the initial choice of the set of numbers $l_{i}$ in (5.1), we obtain either some isotonic oscillator system or some isospectral deformation. If, however, we do not eliminate in such a way all the separated lower lying energy levels in the spectrum of $L^{1 / 2}$, we obtain some nonisospectral, gapped rational extension of some isotonic oscillator. Using the general properties of the DC transformations, the described procedure with two DC transformations can be unified into just one DC transformation based on the corresponding set of physical eigenstates of the quantum harmonic oscillator chosen as seed states.
Equivalently, the chosen complete set of the physical eigenstates of the quantum harmonic oscillator $L^{\text {osc }}$ will correspond to both physical and nonphysical eigenstates of the half-harmonic oscillator $L_{0}$. A set of physical states of $L_{0}$ is formed by the set of odd eigenstates of $L^{\text {osc }}$. The set of nonphysical eigenstates of $L_{0}$ corresponds here to the even eigenstates of $L^{\text {osc }}$; they are formal eigenstates of $L_{0}$ that do not satisfy the boundary condition for the halfharmonic oscillator at $x=0$.

Let us first consider a simple example taking $m=1$ and fixing $l_{1}=4$. According to (5.1), we obtain the system $L_{(4,5)}$ that is a deformed harmonic oscillator with a gap of size six and four separated states given by the corresponding DC map of the states $\psi_{0}, \psi_{1}, \psi_{2}$, and $\psi_{3}$. Following the general prescription described above, we introduce an infinite barrier at $x=0$ to produce the halved deformed harmonic oscillator $L_{(4,5)}^{1 / 2}$ in which all the states with an even index are removed and the two lowest states are separated from the equidistant part of the spectrum by a gap of size eight. Then we use the ground state of this system, which is given by the image of the state $\psi_{1}$, to generate finally the deformed isotonic oscillator $L_{(1,4,5)}$ with a potential $V_{(1,4,5)}$ given by Eq. (D2). The system has one separated state and the size of the gap is equal to eight. This is the minimal possible size for a gap in the spectrum that can be created by the described general procedure. The dual scheme is $(-2,-3,-5) \sim(1,4,5)$, and we can reinterpret both these schemes in terms of the isotonic oscillator. For this we generate the system $L_{1}$ by eliminating the ground state $\psi_{1}$ of the half-harmonic oscillator and then use the second excited state of this system given by $A_{1}^{-} \psi_{5}(x)$ together with the nonphysical state $A_{1}^{-} \psi_{4}(x)$, which is singular at zero. The use of both indicated states allows us to generate a system to be regular on the positive half-line. In a similar way we also can reinterpret the negative scheme by generating the system $L_{3}$ with the help of the auxiliary scheme $(-1,-3,-5)$. Then we apply to it additional DC transformation with the seed states $\mathbb{A}_{-3}^{-} \widetilde{\psi_{1}^{-}}=\mathcal{L}_{2}^{(-7 / 2)}\left(x^{2}\right) x^{-3} e^{-x^{2} / 2}$ and $\mathbb{A}_{-3}^{-} \psi_{2}^{-}=$ $\mathcal{L}_{1}^{(-7 / 2)}\left(-x^{2}\right) x^{-3} e^{x^{2} / 2}$, where $\mathcal{L}_{2}^{(-7 / 2)}\left(x^{2}\right)$ has no zeros on the positive half-line $x>0$, and $\mathcal{L}_{1}^{(-7 / 2)}\left(-x^{2}\right)$ has one zero there. The first state can be obtained by applying the transformation $m \rightarrow-(m+1)$ with $m=3$ to the second exited state of $L_{3}$, while the second state is generated by applying the same transformation to the first excited state with subsequent change $x \rightarrow i x$. As a result, we produce the same, up to a displacement of the potential for an additive constant, final system. If we look step by step, in this last scheme the image of $\widetilde{\psi_{1}^{-}}$generates an isospectral deformation, under which the image of $\psi_{2}^{-}$, given by

$$
\phi(x)=\frac{W\left(\mathbb{A}_{-3}^{-} \widetilde{\psi_{1}^{-}}, \mathbb{A}_{-3}^{-} \psi_{2}^{-}\right)}{\mathbb{A}_{-3}^{-} \widetilde{\psi_{1}^{-}}}=e^{x^{2} / 2} \frac{15+10 x^{2}-4 x^{4}+8 x^{6}}{x^{2}\left(15+4 x^{2}\left(3+x^{2}\right)\right)},
$$


deforms additionally the potential by introducing a new ground state $1 / \phi(x)$.

It is worthwhile to note that we can construct another system to be completely isospectral to $L_{(1,4,5)}$ by taking the schemes $(1,5,6) \sim(-2,-3,-4,-6)$ [that corresponds to $m=1$ and $l_{1}=5$ in Eq. (5.1)], whose potential is given by Eq. (D3). As we shall see below, the dual schemes with even the highest even index in the positive scheme have some peculiarity in the construction of the ladder operators.

In order to see how the described procedure works in the general case, and to reveal and discuss the peculiarities related to the construction and nature of the ladder operators, we consider a more complicated concrete nontrivial example. Let us take $m=2$ and choose $l_{1}=4$ and $l_{2}=10$ in (5.1). We then obtain the rationally extended quantum harmonic oscillator system described by the Hamiltonian operator $L_{(4,5,10,11)}=-\frac{d^{2}}{d x^{2}}+x^{2}-$ $2(\ln W(4,5,10,11))^{\prime \prime}$. The ground state in the halved system $L_{(4,5,10,11)}^{1 / 2}$ corresponds to the DC transformation of the eigenstate $\psi_{1}$ of the quantum harmonic oscillator that has the form $\psi=W(4,5,10,11,1) / W(4,5,10,11)$. Realizing the Darboux transformation of $L_{(4,5,10,11)}^{1 / 2}$ on the basis of this $\psi$ chosen as the seed state, we obtain the system on the half-line that equivalently can be presented by the Hamiltonian operator

$L_{(1,4,5,10,11)}=-\frac{d^{2}}{d x^{2}}+x^{2}-2(\ln W(1,4,5,10,11))^{\prime \prime}$.

This Hamiltonian corresponds to the DC transformation of the half-harmonic oscillator $L_{0}$ generated on the basis of the set of its physical and nonphysical eigenstates $(1,4,5,10,11)$. The system (5.3) represents a nonisospectral rational extension of the isotonic oscillator system $L_{1}$. The explicit analytical form of the potential is given by Eq. (D4). The graph of this potential and the quantum spectrum of the system (5.3) are shown in Fig. 2. The potential has three local minima and the system supports three separated states in its spectrum that are organized in two valence bands of one and two states. By the method of the mirror diagram, we find that the DC scheme $(-2,-3,-4,-5,-8,-9,-11)$ produces the same Hamiltonian operator but shifted by a constant, $L_{(+)}-$ $L_{(-)}=6 \Delta E^{\text {iso }}=24$; see Fig. 1 . Here, for simplicity, we introduced the notations $L_{(+)}=L_{(1,4,5,10,11)}$ and $L_{(-)}=$ $L_{(-2,-3,-4,-5,-8,-9,-11)}$. The fact that the mutual shift of both Hamiltonians is proportional to the difference of two consecutive energy levels in the spectrum of the isotonic oscillator allows us to use below exactly the same rule for the construction of the ladder operators of the type $\mathcal{C}^{ \pm}$as in the previous section. As we shall see, the number of physical states annihilated by the lowering operator $\mathcal{C}^{-}$

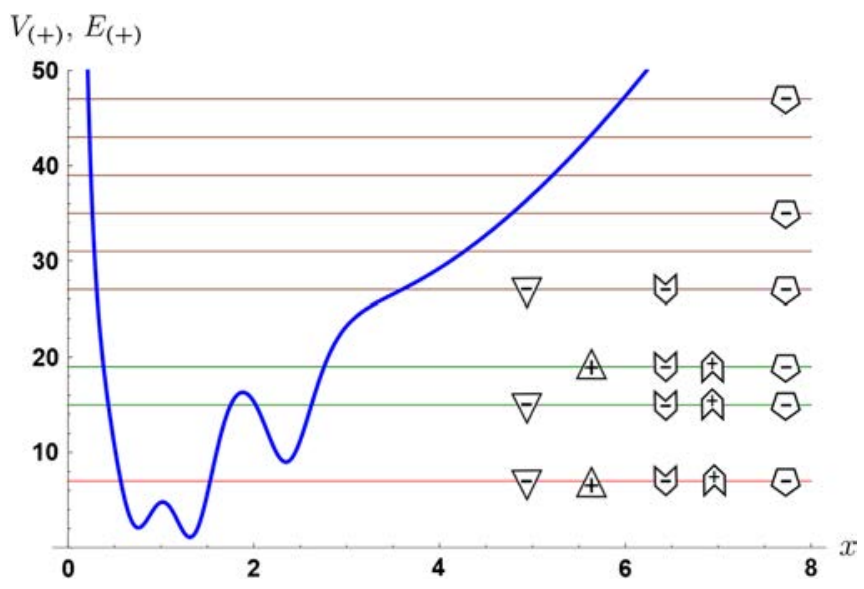

FIG. 2. Potential (D4) of the system (5.3). The energy levels of the corresponding physical states annihilated by ladder operators $\mathcal{B}^{-}, \mathcal{B}^{+}, \mathcal{A}^{-}, \mathcal{A}^{+}$, and $\mathcal{C}^{-}$are indicated from left to right.

in this case is equal exactly to six. Later, we also shall see that in some cases of the rational gapped deformations of the isotonic oscillator systems, the mutual shift of the corresponding Hamiltonian operators can be equal to the half-integer multiple of $\Delta E^{\text {iso }}$, and then the procedure for the construction of the ladder operators of the type $\mathcal{C}^{ \pm}$will require some modification.

In the DC construction of the Hamiltonian operator $L_{(+)}$, the energy levels corresponding to the physical seed eigenstates of the half-harmonic oscillator $L_{0}$ were removed from the spectrum producing two gaps. In the equivalent system $L_{(-)}$based on nonphysical seed eigenstates of $L_{0}$, the energy levels were added under the lowest energy of the ground state of $L_{0}$. The fifth order differential operators that intertwine the half-harmonic oscillator $L_{0}$ with $L_{(+)}$are

$$
\mathbb{A}_{(+)}^{-}=\mathbb{A}_{(1,4,5,10,11)}^{-}, \quad \mathbb{A}_{(+)}^{+}=\mathbb{A}_{(1,4,5,10,11)}^{+} .
$$

The seventh order differential operators intertwining $L_{0}$ with $L_{(-)}$are

$$
\begin{aligned}
& \mathbb{A}_{(-)}^{-}=\mathbb{A}_{(-2,-3,-4,-5,-8,-9,-11)}^{-}, \\
& \mathbb{A}_{(-)}^{+}=\mathbb{A}_{(-2,-3,-4,-5,-8,-9,-11)}^{+} .
\end{aligned}
$$

The three lowest physical states of the system (5.3) which correspond to the three separated energy levels can be presented in two equivalent forms $\mathbb{A}_{(-)}^{-} \widetilde{\psi_{8}^{-}}=\mathbb{A}_{(+)}^{-} \psi_{3}$, $\mathbb{A}_{(-)}^{-} \widetilde{\psi_{4}^{-}}=\mathbb{A}_{(+)}^{-} \psi_{7}, \quad \mathbb{A}_{(-)}^{-} \widetilde{\psi_{2}^{-}}=\mathbb{A}_{(+)}^{-} \psi_{9}$, where equalities are modulo a nonzero constant multiplier. We have here the intertwining relations

$$
\begin{aligned}
& \mathbb{A}_{(+)}^{-} L_{0}=L_{(+)} \mathbb{A}_{(+)}^{-}=\left(L_{(-)}+24\right) \mathbb{A}_{(+)}^{-}, \\
& \mathbb{A}_{(-)}^{-} L_{0}=L_{(-)} \mathbb{A}_{(-)}^{-}=\left(L_{(+)}-24\right) \mathbb{A}_{(-)}^{-},
\end{aligned}
$$

and the conjugate relations for $\mathbb{A}_{(+)}^{+}$and $\mathbb{A}_{(-)}^{+}$. 
From the point of view of the isotonic oscillator we can reinterpret the positive scheme starting from $L_{1}$. One can check that the state $A_{1}^{-} \psi_{4}=x^{-1} \mathcal{L}_{2}^{(-3 / 2)}\left(x^{2}\right) e^{-x^{2} / 2}$ is obtainable by applying the transformation $m \rightarrow-m-1$ with $m=1$ to the second excited state while $A_{1}^{-} \psi_{10}=$ $x^{-1} \mathcal{L}_{5}^{(-3 / 2)}\left(x^{2}\right) e^{-x^{2} / 2}$ is generated by the same transformation from the fifth excited state. By iterative properties of the DC transformations we can see that the selection of the states $\left\{A_{1}^{-} \psi_{4}, A_{1}^{-} \psi_{10}, A_{1}^{-} \psi_{5}, A_{1}^{-} \psi_{11}\right\}$ produces our deformed system. The negative scheme here can also be reinterpreted by constructing first the isotonic system $L_{6}$ by using the set of six odd nonphysical states $\{-1,-3,-5,-7,-9,-11\}$ and then taking $\mathbb{A}_{-6}^{-} \widetilde{\psi_{1}^{-}}$, $\mathbb{A}_{-6}^{-} \widetilde{\psi_{7}^{-}}, \mathbb{A}_{-6}^{-} \psi_{2}^{-}, \mathbb{A}_{-6}^{-} \psi_{4}^{-}$, and $\mathbb{A}_{-6}^{-} \psi_{8}^{-}$as the seed states. From the point of view of $L_{6}$, these states can be obtained from some physical states by applying the symmetry transformations of the isotonic oscillator as in the previous considered cases, and if we trace step by step, we see that the states $\mathbb{A}_{-6}^{-} \widetilde{\psi_{1}^{-}}$and $\mathbb{A}_{-6}^{-} \widetilde{\psi_{7}^{-}}$produce some isospectral deformation of $L_{4}$. In this new intermediate system three other states introduce three separated states below the equidistant part of the spectrum by producing finally our deformed oscillator system (5.3).

Let us turn now to the construction of the ladder operators for the system under consideration. As in the previous section, we begin with the Darboux-dressed ladder operators, and we first consider the operators of the type $\tilde{\mathcal{B}}^{ \pm}$. They are constructed from the ladder operators $\mathcal{C}_{1}^{ \pm}$of the isotonic oscillator $L_{1}$, and are the lowest (tenth) order ladder operators we can obtain by using the method of Darboux dressing:

$$
\tilde{\mathcal{B}}^{ \pm}=\mathbb{A}_{(4,5,10,11)}^{(1)-} \mathcal{C}_{1}^{ \pm} \mathbb{A}_{(4,5,10,11)}^{(1)+}, \quad\left[L_{(+)}, \tilde{\mathcal{B}}^{ \pm}\right]= \pm \Delta E^{\text {iso } \tilde{\mathcal{B}}^{ \pm}}
$$

The fourth order differential operators $\mathbb{A}_{(4,5,10,11)}^{(1) \pm}$ intertwine the Hamiltonian operators $L_{1}$ and $L_{(+)}$and realize the mapping between their corresponding eigenstates. The numbers in the subscript indicate the eigenstates of the harmonic oscillator used as the seed states of the DC transformation, and the superscript marks the system to which those states are mapped. In correspondence with this notation and properties of the DC transformations, we have here the equality $\mathbb{A}_{(4,5,10,11)}^{(1)-}\left(A_{(1)}^{-} \psi\right)=\mathbb{A}_{(1,4,5,10,11)}^{-} \psi$, where $\psi$ is an arbitrary eigenstate of the half-harmonic oscillator Hamiltonian $L_{0}$. The kernel of $\tilde{\mathcal{B}}^{-}$can be identified by calculating the product $\tilde{\mathcal{B}}^{+} \tilde{\mathcal{B}}^{-}$, while the kernel of $\tilde{\mathcal{B}}^{+}$is found from the product $\tilde{\mathcal{B}}^{-} \tilde{\mathcal{B}}^{+}$. We find

$$
\begin{gathered}
\operatorname{ker} \tilde{\mathcal{B}}^{-}=\operatorname{span}\left\{\mathbb{A}_{(-)}^{-} \widetilde{\psi_{11}^{-}}, \mathbb{A}_{(-)}^{-} \widetilde{\psi_{8}^{-}}, \mathbb{A}_{(-)}^{-} \psi_{7}^{-}, \mathbb{A}_{(-)}^{-} \psi_{6}^{-}, \mathbb{A}_{(-)}^{-} \widetilde{\psi_{5}^{-}},\right. \\
\left.\mathbb{A}_{(-)}^{-} \widetilde{\psi_{4}^{-}}, \mathbb{A}_{(-)}^{-} \psi_{1}^{-}, \mathbb{A}_{(-)}^{-} \psi_{0}^{-}, \mathbb{A}_{(-)}^{-} \psi_{0}, \mathbb{A}_{(-)}^{-} \psi_{1}\right\}
\end{gathered}
$$

The kernel of $\tilde{\mathcal{B}}^{-}$contains three physical states $\mathbb{A}_{(-)}^{-} \widetilde{\psi_{8}^{-}}$, $\mathbb{A}_{(-)}^{-} \widetilde{\psi_{4}^{-}}$, and $\mathbb{A}_{(-)}^{-} \psi_{1}=\mathbb{A}_{(+)}^{-} \psi_{13}$. These are the ground state, the lower state in the two-states valence band (that is also the first excited state of the system), and the lowest state in the equidistant part of the spectrum; see Fig. 2. They are eigenstates of $L_{(-)}$of energies $-17,-9$, and 3, respectively. The kernel

$$
\begin{array}{r}
\operatorname{ker} \tilde{\mathcal{B}}^{+}=\operatorname{span}\left\{\mathbb{A}_{(-)}^{-} \psi_{13}^{-}, \mathbb{A}_{(-)}^{-} \psi_{10}^{-}, \mathbb{A}_{(-)}^{-} \widetilde{\psi_{9}^{-}}, \mathbb{A}_{(-)}^{-} \widetilde{\psi_{8}^{-}}, \mathbb{A}_{(-)}^{-} \psi_{7}^{-},\right. \\
\left.\mathbb{A}_{(-)}^{-} \psi_{6}^{-}, \mathbb{A}_{(-)}^{-} \widetilde{\psi_{3}^{-}}, \mathbb{A}_{(-)}^{-} \widetilde{\psi_{2}^{-}}, \mathbb{A}_{(-)}^{-} \psi_{1}^{-}, \mathbb{A}_{(-)}^{-} \psi_{0}^{-}\right\}
\end{array}
$$

contains two physical states $\mathbb{A}_{(-)}^{-} \widetilde{\psi_{8}^{-}}$and $\mathbb{A}_{(-)}^{-} \widetilde{\psi_{2}^{-}}$, which are identified as the ground state and the second excited state of the system. Both states are the upper states in the one-state and the two-states valence bands. This is correlated with the property that the jump produced by the raising operator $\tilde{\mathcal{B}}^{+}$ is not sufficient to bridge the corresponding gaps in the spectrum. The operators $\tilde{\mathcal{B}}^{ \pm}$cannot connect the part of the spectrum corresponding to the separated states with the states in the equidistant part of the spectrum. Also, they do not connect the states from different valence bands. These ladder operators do not form the set of the spectrumgenerating operators since they do not allow us to generate an arbitrary state in the spectrum from any other arbitrarily chosen fixed physical eigenstate. All the space of the states of the system is separated into three bands, in each of which the operators $\tilde{\mathcal{B}}^{ \pm}$act irreducibly. These ladder operators commute for a certain polynomial of order nine in the Hamiltonian $L_{(-)}$.

As in the isospectral case considered in the previous section, here we have two ways to realize Darboux dressing of the ladder operators $-\mathcal{C}_{0}^{ \pm}=\left(a^{ \pm}\right)^{2}$ of the half-harmonic oscillator system $L_{0}$. Using for this purpose the intertwining operators $\mathbb{A}_{(+)}^{ \pm}$, we obtain differential operators of order 12:

$\mathcal{B}^{ \pm}=\mathbb{A}_{(+)}^{-}\left(a^{ \pm}\right)^{2} \mathbb{A}_{(+)}^{+}, \quad\left[L_{(-)}, \mathcal{B}^{ \pm}\right]= \pm \Delta E^{\text {iso }} \mathcal{B}^{ \pm}$.

With the help of the product $\mathcal{B}^{+} \mathcal{B}^{-}=\left(L_{(-)}+21\right)^{2} \tilde{\mathcal{B}}^{+} \tilde{\mathcal{B}}^{-}$, we find the kernel of the operator $\mathcal{B}^{-}$: $\operatorname{ker} \mathcal{B}^{-}=$ $\operatorname{span}\left\{\mathbb{A}_{(-)}^{-} \psi_{10}^{-}, \mathbb{A}_{(-)}^{-} \widetilde{\psi_{10}^{-}}, \operatorname{ker} \tilde{\mathcal{B}}^{-}\right\}$. The first two states in the kernel of $\mathcal{B}^{-}$are nonphysical eigenstates of the operator $L_{(-)}$of energy $E_{(-)}=-21$. As a result, we see that the ladder operator $\mathcal{B}^{-}$annihilates exactly the same three physical eigenstates as the ladder operator $\tilde{\mathcal{B}}^{-}$, and the other nine eigenstates from the kernel of $\mathcal{B}^{-}$are nonphysical. From here one can suspect that the operators $\mathcal{B}^{ \pm}$and $\tilde{\mathcal{B}}^{ \pm}$are related in a simple way, and we find

$\mathcal{B}^{-}=\tilde{\mathcal{B}}^{-}\left(L_{(-)}+21\right), \quad \mathcal{B}^{+}=\left(L_{(-)}+21\right) \tilde{\mathcal{B}}^{+}$. 
The kernel of $\mathcal{B}^{+}$is spanned by the kernel of $\tilde{\mathcal{B}}^{+}$and two nonphysical eigenstates $\mathbb{A}_{(-)}^{-} \psi_{12}^{-}$and $\mathbb{A}_{(-)}^{-} \widetilde{\psi_{12}^{-}}$of $L_{(-)}$of eigenvalue $E_{(-)}=-25$.

We also can construct the ladder operators by using the intertwining operators $\mathbb{A}_{(-)}^{ \pm}$,

$$
\mathcal{A}^{ \pm}=\mathbb{A}_{(-)}^{-}\left(a^{ \pm}\right)^{2} \mathbb{A}_{(-)}^{+}, \quad\left[L_{(+)}, \mathcal{A}^{ \pm}\right]= \pm \Delta E^{\text {iso }} \mathcal{A}^{ \pm} .
$$

These are also not spectrum-generating operators because the leap they make does not allow them to overcome the gaps. On the other hand, since these are differential operators of order 16, by analogy with the case of rationally extended quantum harmonic oscillator systems [45], one could expect that they annihilate more physical states in comparison with the ladder operators $\mathcal{B}^{ \pm}$and $\tilde{\mathcal{B}}^{ \pm}$. To identify the kernels of the operators $\mathcal{A}^{ \pm}$, it is more convenient to work with the operator $L_{(+)}$instead of $L_{(-)}$. By calculating the alternative products of the operators $\mathcal{A}^{ \pm}$, one can see that they can be written in terms of $\tilde{\mathcal{B}}^{ \pm}$:

$\mathcal{A}^{-}=\tilde{\mathcal{B}}^{-}\left(L_{(+)}-5\right)\left(L_{(+)}-17\right)\left(L_{(+)}-19\right)$,

$\mathcal{A}^{+}=\left(\mathcal{A}^{-}\right)^{\dagger}$.

From here one finds that the raising operator $\mathcal{A}^{+}$detects all the states in both separated valence bands by annihilating them. In addition to the indicated physical states, the lowering operator $\mathcal{A}^{-}$also annihilates the lowest state in the half-infinite equidistant part of the spectrum.

Therefore, the essential difference of the nonisospectral rational deformations of the isotonic oscillator systems from their isospectral rational extensions is that there is no pair of spectrum-generating ladder operators constructed via the Darboux-dressing procedure. This situation is similar to that in the rationally extended quantum harmonic oscillator systems [45].

We now construct the ladder operators $\mathcal{C}^{ \pm}$by "gluing" the intertwining operators of different types. As in the case of the isospectral deformations, they also will not be the spectrum-generating operators, but together with any pair of the ladder operators $\tilde{\mathcal{B}}^{ \pm}, \mathcal{B}^{ \pm}$, or $\mathcal{A}^{ \pm}$they will form the set of the spectrum-generating operators. So, let us consider the differential operators of order 12 ,

$$
\begin{aligned}
\mathcal{C}^{-} & =\mathbb{A}_{(-)}^{-} \mathbb{A}_{(+)}^{+}, \quad \mathcal{C}^{+}=\mathbb{A}_{(+)}^{-} \mathbb{A}_{(-)}^{+}, \\
{\left[L_{(-)}, \mathcal{C}^{ \pm}\right] } & = \pm 6 \Delta E^{\mathrm{iso}} \mathcal{C}^{ \pm} .
\end{aligned}
$$

They are independent from the ladder operators constructed via the Darboux-dressing procedure, and their commutator $\left[\mathcal{C}^{-}, \mathcal{C}^{+}\right]$is a certain polynomal of order 11 in the Hamiltonian $L_{(-)}$. The operators $\mathcal{C}^{ \pm}$divide the space of the states of the system into six infinite subsets on which they act irreducibly. The same number six here also corresponds to the number of physical states $\mathbb{A}_{(-)}^{-} \widetilde{\psi_{8}^{-}}, \mathbb{A}_{(-)}^{-} \widetilde{\psi_{4}^{-}}, \mathbb{A}_{(-)}^{-} \widetilde{\psi_{2}^{-}}$, $\mathbb{A}_{(-)}^{-} \psi_{1}, \mathbb{A}_{(-)}^{-} \psi_{5}, \mathbb{A}_{(-)}^{-} \psi_{11}$ annihilated by the lowering operator $\mathcal{C}^{-}$. The operator $\mathcal{C}^{-}$transforms a physical eigenstate into another physical eigenstate by lowering its level by six. If the arrival level is not in the spectrum, then that state is annihilated by $\mathcal{C}^{-}$.

The operator $\mathcal{C}^{+}$does not annihilate any physical state here. Like $\mathcal{C}^{-}$, it connects the separated states with the equidistant part of the spectrum. As a result, the pair of the ladder operators $\mathcal{C}^{ \pm}$together with any pair of the ladder operators $\tilde{\mathcal{B}}^{ \pm}, \mathcal{B}^{ \pm}$, or $\mathcal{A}^{ \pm}$allows us to transform any physical state into any physical state. For instance, the separated ground state $\mathbb{A}_{(-)}^{-} \widetilde{\psi_{8}^{-}}$of the system can be transformed into the second state $\mathbb{A}_{(-)}^{-} \widetilde{\psi_{2}^{-}}$in the second valence band by application of the product of the ladder operators $\mathcal{C}^{-}\left(\tilde{\mathcal{B}}^{+}\right)^{3} \mathcal{C}^{+}$. Then, the ground state can be transformed into the first excited state $\mathbb{A}_{(-)}^{-} \widetilde{\psi_{4}^{-}}$, being the lowest state in the two-states valence band, by the operator $\tilde{\mathcal{B}}^{-} \mathcal{C}^{-}\left(\tilde{\mathcal{B}}^{+}\right)^{3} \mathcal{C}^{+}$. The ground state can also be transformed into the lowest state of the two-states valence band by the operator $\mathcal{C}^{-}\left(\mathcal{A}^{+}\right)^{2} \mathcal{C}^{+}$. The application of the operator $\mathcal{B}^{-} \mathcal{C}^{+}$ to the ground state transforms it into the lowest state $\mathbb{A}_{(-)}^{-} \psi_{1}$ of the equidistant part of the spectrum. So, the indicated pairs of the ladder operators form the sets of the spectrumgenerating operators of the system. Figure 3 illustrates the action of the ladder operators. Now, consider briefly the systems that can be obtained from the system we have just described by taking its separated states as the seed states to generate the corresponding new DC transformations. Each time, the incorporation of a separated state into a set of the seed states deforms the potential and decreases in one the number of its local minima. As a result, this reduces in one the number of separated states in the spectrum of the corresponding system.

If in the set of the seed states for the DC transformation we include additionally the first excited state of the halved rationally extended quantum harmonic oscillator system $L_{(4,5,10,11)}^{1 / 2}$, or, in an equivalent way, if we realize a Darboux transformation of the system $L_{(1,4,5,10,11)}$ by using its lowest physical state $\mathbb{A}_{(+)}^{-} \psi_{3}=\mathbb{A}_{(-)}^{-} \widetilde{\psi_{8}^{-}}$from the one-state valence band, we obtain the system $L_{(1,3,4,5,10,11)}$. It corresponds to a nonisospectral deformation of the isotonic oscillator $L_{2}$ with the spectrum containing two separated states organized into one two-state valence band. A dual scheme is $(-2,-3,-4,-5,-9,-11) \sim(1,3,4,5,10,11)$. As before, we simplify notations by denoting $L_{(+)}=L_{(1,3,4,5,10,11)}$ and $L_{(-)}=L_{(-2,-3,-4,-5,-9,-11)}$, for which, again, $L_{(+)}-$ $L_{(-)}=24$. The sixth order differential operators that intertwine $L_{(+)}$and $L_{(-)}$with $L_{0}$ are $\mathbb{A}_{(+)}^{ \pm}=\mathbb{A}_{(1,3,4,5,10,11)}^{ \pm}$and $\mathbb{A}_{(-)}^{ \pm}=\mathbb{A}_{(-2,-3,-4,-5,-9,-11)}^{ \pm}$. With their help we construct 

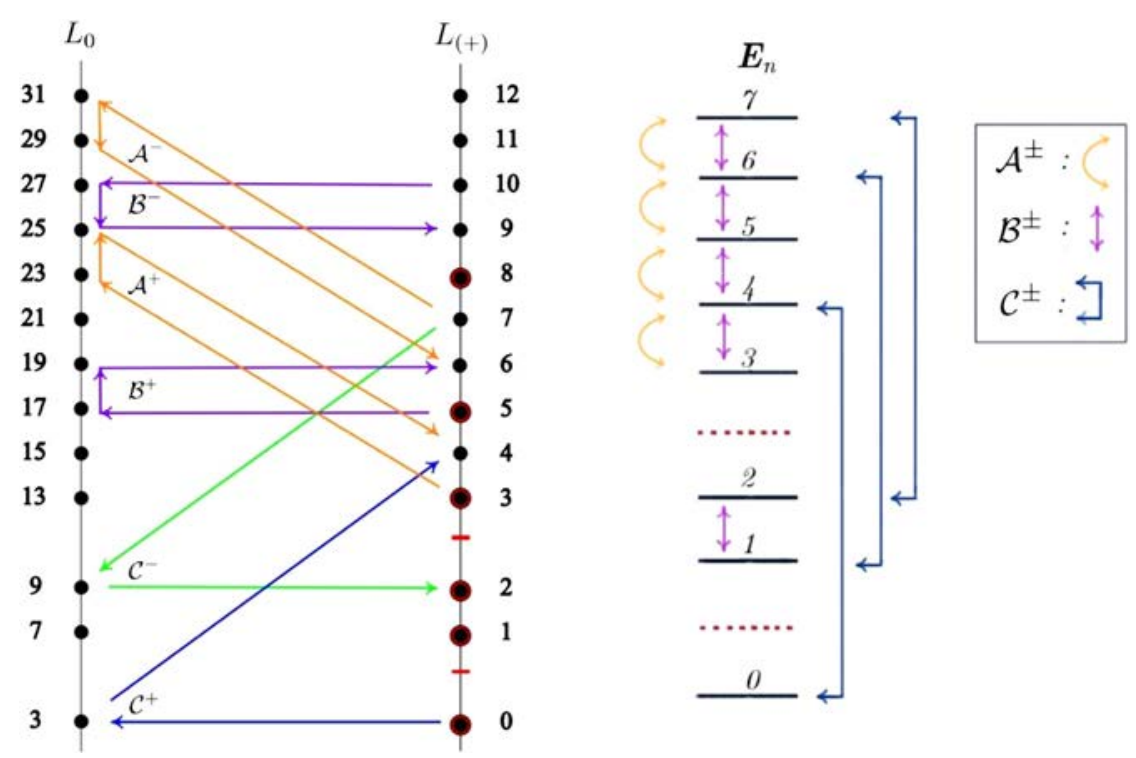

FIG. 3. Left panel: The numbers on the left correspond to the indices of the physical eigenstates $\psi_{2 l+1}$ of the half-harmonic oscillator that are mapped "horizontally" by operator $\mathbb{A}_{(+)}^{-}$into eigenstates $\Psi_{n}$ of the system (5.3). Lines show the action of the ladder operators coherently with their structure (5.12), (5.10), and (5.14). The marked set of the states $0,1,2,3,5,8$ on the right corresponds to six eigenstates of $L_{(+)}$annihilated by $\mathcal{C}^{-}$. Right panel: Horizontal lines correspond to the energy levels of $L_{(+)}$. Upward and downward arrows represent the action of the rising and lowering ladder operators, respectively. Following appropriate paths, any eigenstate can be transformed into any other eigenstate by applying subsequently the corresponding ladder operators.

the ladder operators $\mathcal{B}^{ \pm}$and $\mathcal{A}^{ \pm}$by the DC dressing of the ladder operators $-\mathcal{C}_{0}^{ \pm}=\left(a^{ \pm}\right)^{2}$ of $L_{0}$ as it was done for the system (5.3). The order 12 ladder operators $\mathcal{C}^{ \pm}$are also constructed in the form (5.14) using the sixth order intertwining operators $\mathbb{A}_{(+)}^{ \pm}$and $\mathbb{A}_{(-)}^{ \pm}$. The analogs of the ladder operators (5.7) are constructed here by the DC dressing of the ladder operators of the isotonic oscillator $L_{2}, \quad \tilde{\mathcal{B}}^{ \pm}=\mathbb{A}_{(4,5,10,11)}^{(1,3)-} \mathcal{C}_{2}^{ \pm} \mathbb{A}_{(4,5,10,11)}^{(1,3)+} . \quad$ The $\mathbb{A}_{(4,5,10,11)}^{(1,3)-}$ and $\mathbb{A}_{(4,5,10,11)}^{(1,3)+}=\left(\mathbb{A}_{(4,5,10,11)}^{(1,3)-}\right)^{\dagger}$ are the fourth order operators intertwining the system $L_{(1,3,4,5,10,11)}$ with the isotonic oscillator $L_{2}$. They are given by relations of the form

$$
\begin{aligned}
\mathbb{A}_{(4,5,10,11)}^{(1,3)-}\left(\mathbb{A}_{(1,3)}^{-} \psi\right) & =\mathbb{A}_{\left(\mathbb{A}_{2}^{-} 4, \mathbb{A}_{2}^{-} 5, \mathbb{A}_{2}^{-} 10, \mathbb{A}_{2}^{-} 11\right)}^{-}\left(\mathbb{A}_{2}^{-} \psi\right) \\
& =\mathbb{A}_{(1,3,4,5,10,11)}^{-} \psi
\end{aligned}
$$

where $\mathbb{A}_{(1,3)}^{-}=\mathbb{A}_{2}^{-}$is the operator (2.11) with $m=2$ that intertwines $L_{0}$ and $L_{2}$. The ladder operators $\mathcal{B}^{ \pm}$and $\tilde{\mathcal{B}}^{ \pm}$act on physical states in the same way. The lowering operators annihilate the ground state being the lower state in the twostates valence band and the lowest state in the equidistant part of the spectrum. The raising operators annihilate the first excited state that corresponds to the upper energy level in the valence band. The raising operator $\mathcal{A}^{+}$annihilates both states in the valence band of energies $E_{(+)}=15$ and $E_{(+)}=19$, whereas the lowering operator $\mathcal{A}^{-}$annihilates in addition the lowest state of energy $E_{(+)}=27$ in the equidistant part of the spectrum. One can find the relations between these ladder operators as it was done for the system (5.3). The lowering operator $\mathcal{C}^{-}$annihilates both states $\mathbb{A}_{(+)}^{-} \psi_{7}$ and $\mathbb{A}_{(+)}^{-} \psi_{9}$ of the valence band, the lowest state $\mathbb{A}_{(+)}^{-} \psi_{13}$ in the equidistant part of the spectrum, and three more physical states there which are $\mathbb{A}_{(+)}^{-} \psi_{15}, \mathbb{A}_{(+)}^{-} \psi_{17}$, and $\mathbb{A}_{(+)}^{-} \psi_{23}$ of energies $E_{(+)}=31$, $E_{(+)}=35$, and $E_{(+)}=47$. Besides, it also annihilates six nonphysical eigenstates. The kernel of the raising operator $\mathcal{C}^{+}$contains only nonphysical states. As in the previous system $L_{(1,4,5,10,11)}$, the ladder operators $\mathcal{C}^{ \pm}$ together with any of the pairs of ladder operators $\tilde{\mathcal{B}}^{ \pm}$, $\mathcal{B}^{ \pm}$, or $\mathcal{A}^{ \pm}$form the sets of the spectrum-generating operators of the system $L_{(1,3,4,5,10,11)}$.

One can continue in the same vein and obtain the system $L_{(1,3,4,5,7,10,11)}=L_{(-2,-3,-5,-9,-11)}+24$ that has the same spectrum as the system $L_{(1,4,5,10,11)}$ but with the omitted two lowest energy levels. This is a nonisospectral rational deformation of the isotonic oscillator $L_{3}$ with one energy level separated by a distance $2 \Delta E^{\text {iso }}=8$ from the equidistant part of the spectrum. Finally, we obtain the system $L_{(1,3,4,5,7,9,10,11)}=L_{(-3,-5,-9,-11)}+24$ by eliminating the unique separated energy level from the spectrum of the system $L_{(1,3,4,5,7,9,10,11)}$. This is an isospectral deformation of the isotonic oscillator $L_{4}$.

All of the described picture is generalized directly in the case when the index of the last seed state used in the corresponding DC transformation is odd. Then the 
corresponding scheme based on physical eigenstates of $L_{0}$ is of the form $\left(\ldots, 2 l_{m}, 2 l_{m}+1\right)$, and the dual scheme is $\left(\ldots,-\left(2 l_{m}+1\right)\right)$. Following the same notation as we used in the particular examples, the Hamiltonian operators generated in these two dual schemes are shifted by the distance equal to the separation $\Delta E^{\text {iso }}=4$ of energy levels in the equidistant part of the spectrum times integer number $l_{m}+1: L_{(+)}-L_{(-)}=4 l_{m}+4$. The operators $\mathbb{A}_{(+)}^{ \pm}$that intertwine the Hamiltonian $L_{(+)}$with $L_{0}$,

$$
\mathbb{A}_{(+)}^{-} L_{0}=L_{(+)} \mathbb{A}_{(+)}^{-}=\left(L_{(-)}+4 l_{m}+4\right) \mathbb{A}_{(+)}^{-},
$$

are of differential order $n_{+}$, where $n_{+}$corresponds to the number of seed states in the positive scheme $\left(\ldots, 2 l_{m}, 2 l_{m}+1\right)$. The operators $\mathbb{A}_{(-)}^{ \pm}$intertwining $L_{(-)}$ with $L_{0}$,

$$
\mathbb{A}_{(-)}^{-} L_{0}=L_{(-)} \mathbb{A}_{(-)}^{-}=\left(L_{(+)}-4 l_{m}-4\right) \mathbb{A}_{(-)}^{-},
$$

in this case are of differential order $2 l_{m}+2-n_{+}$. The ladder operators $\mathcal{A}^{ \pm}=\mathbb{A}_{(-)}^{-}\left(a^{ \pm}\right)^{2} \mathbb{A}_{(-)}^{+}$have differential order $2\left(2 l_{m}+2-n_{+}\right)+2$. The ladder operators $\mathcal{B}^{ \pm}$constructed by Darboux dressing of the ladder operators of the half-harmonic oscillator $L_{0}$ by means of intertwining operators $\mathbb{A}_{(+)}^{ \pm}$are of differential order $2 n_{+}+2$. These ladder operators satisfy commutation relations of the form (5.12) and (5.10). The lowering operator $\mathcal{B}^{-}$annihilates all the lowest states in each valence band and the lowest state in the equidistant part of the spectrum. The raising operator $\mathcal{B}^{+}$annihilates all the highest states in each valence band. The raising operator $\mathcal{A}^{+}$detects all the states in all the valence bands by annihilating them. The lowering ladder operator $\mathcal{A}^{-}$annihilates in addition to all the separated states the lowest state in the equidistant part of the spectrum. The ladder operators $\mathcal{C}^{ \pm}$of the form (5.14) constructed by gluing intertwining operators from dual schemes have a differential order $2 l_{m}+2$, and commute with Hamiltonian operators of the system as follows:

$$
\left[L_{(-)}, \mathcal{C}^{ \pm}\right]= \pm\left(l_{m}+1\right) \Delta E^{\text {iso }} \mathcal{C}^{ \pm} .
$$

Like the ladder operator $\mathcal{A}^{-}$, the lowering operator $\mathcal{C}^{-}$ annihilates all the states in all the valence bands and the lowest state in the equidistant part of the spectrum. In addition, it annihilates a certain number of excited states in the equidistant part of the spectrum. The total number of the physical states annihilated by $\mathcal{C}^{-}$is equal to $l_{m}+1$ that coincides with the corresponding coefficient in (5.18). The kernel of the raising operator $\mathcal{C}^{+}$contains no physical states. The ladder operators $\mathcal{C}^{ \pm}$together with $\mathcal{A}^{ \pm}$or $\mathcal{B}^{ \pm}$ form the set of spectrum-generating operators. In these sets of spectrum-generating operators, the ladder operators $\mathcal{B}^{ \pm}$can be substituted by the ladder operators $\tilde{\mathcal{B}}^{ \pm}$.
The operators $\tilde{\mathcal{B}}^{ \pm}$are constructed by Darboux dressing the ladder operators $\mathcal{C}_{m}^{ \pm}$in the way we constructed such operators in the considered examples. Here we assume that the corresponding Hamiltonians $L_{(+)}$and $L_{(-)}$describe a certain rational deformation of the isotonic oscillator system $L_{m}$. It may or may not happen that the operators of type $\tilde{\mathcal{B}}^{ \pm}$of lower differential order can be obtained by Darboux dressing of ladder operators of another isotonic oscillator system. This, however, depends on the concrete rational deformation of the isotonic oscillator we have and requires a concrete investigation. With respect to the physical states, the ladder operators $\tilde{\mathcal{B}}^{ \pm}$will have the same properties as the ladder operators $\mathcal{B}^{ \pm}$.

When we have the schemes $\left(\ldots, 2 l_{m}-1,2 l_{m}\right) \sim$ $\left(\ldots,-2 l_{m}\right)$ generating a gapped rational extension of some isotonic oscillator system, the corresponding Hamiltonian operators associated with them are shifted mutually for the distance $L_{(+)}-L_{(-)}=4 l_{m}+2=\left(l_{m}+\right.$ $\left.\frac{1}{2}\right) \Delta E^{\text {iso }}$ that is equal to the half-integer multiple of the energy spacing in the equidistant part of the spectrum and in the valence bands with more than one state. In this case the procedure related to the construction of the ladder operators $\mathcal{A}^{ \pm}, \mathcal{B}^{ \pm}$, and $\tilde{\mathcal{B}}^{ \pm}$and their properties are similar to those in the systems generated by the schemes $\left(\ldots, 2 l_{m}, 2 l_{m}+1\right) \sim\left(\ldots,-\left(2 l_{m}+1\right)\right)$. However, the situation with the construction of the ladder operators of the type $\mathcal{C}^{ \pm}$in this case is essentially different. We still can construct the operators $\mathcal{C}^{ \pm}$of the form (5.14). Such operators will be of odd differential order $2 l_{m}+1$, and their commutation relations with any of the Hamiltonian operators $L_{(+)}$and $L_{(-)}$will be of the form $\left[L, \mathcal{C}^{ \pm}\right]= \pm\left(4 l_{m}+2\right) \mathcal{C}^{ \pm}$. This means that these operators acting on physical eigenstates of $L$ will produce nonphysical eigenstates except the case when the lowering operator $\mathcal{C}^{-}$acts on the states from its kernel. The square of these operators will not have the indicated deficiency and will form together with the ladder operators $\mathcal{A}^{ \pm}, \mathcal{B}^{ \pm}$, or $\tilde{\mathcal{B}}^{ \pm}$the set of the spectrum-generating operators. This picture can be compared with the case of the half-harmonic oscillator $L_{0}$, where the first order differential operators $a^{ \pm}$will have the properties similar to those of the described operators $\mathcal{C}^{ \pm}$. In this case we can, however, modify slightly the construction of the ladder operators of the $\mathcal{C}^{ \pm}$type by taking

$$
\tilde{\mathcal{C}}^{-}=A_{(-)}^{-}\left(a^{-}\right) A_{(+)}^{+}, \quad \tilde{\mathcal{C}}^{+}=A_{(+)}^{-}\left(a^{+}\right) A_{(-)}^{+} .
$$

These ladder operators satisfy the commutation relations $\left[L_{( \pm)}, \tilde{\mathcal{C}}^{ \pm}\right]=4\left(l_{m}+1\right) \tilde{\mathcal{C}}^{ \pm}$, and transform physical states into physical states.

Note that the total number of physical eigenstates from the half-infinite equidistant part of the spectrum which belong to the kernel of the ladder operator $\mathcal{C}^{-}$(or $\tilde{\mathcal{C}}^{-}$) in the case of the system of the type $\left(\ldots, 2 l_{m}+1\right)$ [or $\left.\left(\ldots, 2 l_{m}\right)\right]$ 
can be expressed in terms of the sizes $n_{+}$and $n_{-}$of the corresponding positive and negative dual schemes and the total number $n_{v}$ of the states in all the valence bands of the deformed gapped isotonic oscillator system. If we denote such a number by $n_{\infty}$, we obtain $n_{\infty}=\frac{1}{2}\left(n_{+}+\right.$ $\left.n_{-}\right)-n_{v}$ for the system of the type $\left(\ldots, 2 l_{m}+1\right)$, for which $2 l_{m}+1=n_{+}+n_{-}-1$, see Section III. For the system with a positive scheme of the type $\left(\ldots, 2 l_{m}\right)$, we have $n_{\infty}=\frac{1}{2}\left(n_{+}+n_{-}-1\right)-n_{v}$.

To conclude this section, let us summarize the structure of the nonlinearly deformed conformal symmetry algebras generated by different pairs of the corresponding ladder operators and Hamiltonians of the rationally deformed conformal mechanics systems. The commutators of the ladder operators $\mathcal{A}^{ \pm}, \mathcal{B}^{ \pm}$, and $\mathcal{C}^{ \pm}$with Hamiltonian operators are given, respectively, by Eqs. (5.12), (5.10), and (5.14) with $E^{\text {iso }}=4$. The commutation relations of the form (5.12) also are valid for the case of the isospectral deformations discussed in the previous section. To write down the commutation relations between raising and lowering operators of the same type in a general case, let us introduce the polynomial functions

$$
\begin{aligned}
& P_{n_{+}}(x)=\Pi_{k=1}^{n_{+}}\left(x-2 n_{k}-1\right), \\
& R_{n_{-}}(x)=\Pi_{l=1}^{n_{-}}\left(x+2 n_{l}+1\right),
\end{aligned}
$$

where $n_{k}>0$ are the indices of the corresponding seed states in the positive scheme and $-n_{l}<0$ are the indices of the seed states in the negative scheme. With this notation, we have the relations $\mathbb{A}_{(+)}^{+} \mathbb{A}_{(+)}^{-}=P_{n_{+}}\left(L_{0}\right), \mathbb{A}_{(+)}^{-} \mathbb{A}_{(+)}^{+}=$ $P_{n_{+}}\left(L_{(+)}\right)=P_{n_{+}}\left(L_{(-)}+2\left(n_{-}+n_{+}\right)\right)$, and $\mathbb{A}_{(-)}^{+} \mathbb{A}_{(-)}^{-}=$ $R_{n_{-}}\left(L_{0}\right), \mathbb{A}_{(-)}^{-} \mathbb{A}_{(-)}^{+}=R_{n_{-}}\left(L_{(-)}\right)$. Then we obtain

$$
\left[\mathcal{A}^{-}, \mathcal{A}^{+}\right]=\left.(x+1)(x+3) R_{n_{-}}(x) R_{n_{-}}(x+4)\right|_{x=L_{(-)}-4} ^{x=L_{(-)}},
$$

$$
\left[\mathcal{B}^{-}, \mathcal{B}^{+}\right]=\left.(x+1)(x+3) P_{n_{+}}(x+4) P_{n_{+}}(x)\right|_{x=L_{(-)}+2 N-4} ^{x=L_{(-)}+2 N},
$$

$$
\left[\mathcal{C}^{-}, \mathcal{C}^{+}\right]=\left.R_{n_{-}}(x) P_{n_{+}}(x)\right|_{x=L_{(-)}} ^{x=L_{(-)}+2 N},
$$

where $N=n_{-}+n_{+}$, and relation (5.21) also is valid in the case of isospectral deformations. In the case of the nonisospectral deformations given by the dual schemes $\left(\ldots, 2 l_{m}-1,2 l_{m}\right) \sim\left(\ldots,-2 l_{m}\right)$, the corresponding modified operators (5.19) satisfy the commutation relation

$\left[\tilde{\mathcal{C}}^{-}, \tilde{\mathcal{C}}^{+}\right]=\left.(x+1) R_{n_{-}}(x) P_{n_{+}}(x+2)\right|_{x=L_{(-)}} ^{x=L_{(-)}+2 N-2}$.

Thus, in any rational deformation of the conformal mechanics model we considered, each pair of the conjugate ladder operators of the types $\mathcal{A}^{ \pm}, \mathcal{B}^{ \pm}$, or $\mathcal{C}^{ \pm}$generates a nonlinear deformation of the conformal $\mathfrak{s} \mathfrak{l}(2, \mathbb{R})$ symmetry. The commutation relations between ladder operators of different types of the form $\left[\mathcal{A}^{ \pm}, \mathcal{C}^{ \pm}\right]$, etc., not considered here are model dependent, and taking them into account gives rise naturally to different nonlinearly extended versions of the superconformal $\mathfrak{o} \mathfrak{g} \mathfrak{p}(2 \mid 2)$ symmetry [52].

\section{SUMMARY, DISCUSSION, AND OUTLOOK}

We studied rational deformations of the quantum conformal mechanics model and constructed complete sets of the spectrum-generating ladder operators for them by applying the generalized DCKA transformations to the quantum harmonic oscillator and using the method of the dual positive and negative schemes and mirror diagrams.

The positive scheme is constructed by selecting certain $n_{+}$physical states of the harmonic oscillator as the seed states for generalized DCKA transformation. In the negative scheme we use some of its $n_{-}$nonphysical states obtained from the corresponding eigenstates with positive energies by the transformation $x \rightarrow i x, E \rightarrow-E$. The two schemes are related by a kind of charge conjugation presented by the mirror diagram. They generate the same deformed (or undeformed) conformal mechanics system modulo a nonzero relative displacement of the spectrum defined by a number of the seed states participating in both dual schemes. The set of the seed states in any of the two dual schemes has to be chosen in such a way that the resulting system will be regular on the positive half-line.

We showed that each isospectral deformation of the isotonic oscillator $L_{m}$ can be characterized by the ladder operators $\mathcal{A}^{ \pm}$being differential operators of order $2(m+1)=2\left(n_{-}+1\right)$, which are the second order ladder operators of $L_{m}$ Darboux dressed by the conjugate pair of the intertwining operators of order $m$ of the negative scheme. These operators, as the second order ladder operators of $L_{m}$, form a spectrum-generating set of operators by means of which any state of the deformed system can be transformed into any other state. The picture, however, is completely different in the case of the gapped, nonisospectral, deformations of the isotonic oscillators where it is similar to the picture in rationally extended quantum harmonic oscillator systems [45]. The complete set of the spectrum generating operators is formed in this case by two pairs of the ladder operators $\left(\mathcal{A}^{ \pm}, \mathcal{C}^{ \pm}\right)$or $\left(\mathcal{B}^{ \pm}, \mathcal{C}^{ \pm}\right)$. Here the ladder operators $\mathcal{A}^{ \pm}$are constructed by Darboux dressing of the second order ladder operators $\left(a^{ \pm}\right)^{2}$ of the half-harmonic oscillator $L_{0}$ by the intertwining operators of the negative scheme, while $\mathcal{B}^{ \pm}$are obtained by Darboux dressing of the same operators $\left(a^{ \pm}\right)^{2}$ but by the intertwining operators of the positive scheme. The operators $\mathcal{A}^{ \pm}$and $\mathcal{B}^{ \pm}$act similarly to the usual ladder operators in the equidistant part of the spectrum but their action is different in a separated part. The operators $\mathcal{A}^{ \pm}$detect all the 
separated states just by annihilating each of them. The operators $\mathcal{B}^{ \pm}$detect the number of low-lying separated valence bands in the spectrum where $\mathcal{B}^{-}$acts as a lowering operator in each valence band annihilating there the corresponding lowest state. The raising operator $\mathcal{B}^{+}$annihilates the highest state in each valence band. As a result, in each valence band with $l \geq 1$ states, the action of the operators $\mathcal{B}^{ \pm}$is characterized by the relations $\left(\mathcal{B}^{ \pm}\right)^{l}=0$ typical for spin- $s$ ladder operators with $s=(l-1) / 2$. In dependence on the concrete nonisospectral deformation, sometimes the ladder operators $\tilde{\mathcal{B}}^{ \pm}$of the lower differential order can be constructed by the DC dressing of the ladder operators of some isotonic oscillator $L_{m}$ with $m>0$, but their action on physical eigenstates of the deformed system is essentially the same as the action of $\mathcal{B}^{ \pm}$. From the properties of the ladder operators $\mathcal{A}^{ \pm}$and $\mathcal{B}^{ \pm}$it is clear that they cannot connect the states from different valence bands, and the separated states cannot be connected by them with the states from the equidistant part of the spectrum. "Communication" between the indicated states is provided by the ladder operators $\mathcal{C}^{ \pm}$constructed by gluing the intertwining operators of the positive and negative schemes.

In any rational deformation of the conformal mechanics model we considered, each pair of the conjugate ladder operators $\mathcal{A}^{ \pm}, \mathcal{B}^{ \pm}$, and $\mathcal{C}^{ \pm}$or $\tilde{\mathcal{C}}^{ \pm}$in the case of the systems of the type $\left.\left(\ldots, 2 l_{m}-1,2 l_{m}\right) \sim\left(\ldots,-2 l_{m}\right)\right]$ generates some nonlinear deformation of the conformal $\mathfrak{g l}(2, \mathbb{R})$ symmetry with a polynomial of order, respectively, $2 n_{-}-1,2 n_{+}-1$ and $n_{-}+n_{+}-1$ (or $n_{-}+n_{+}+1$ ) in the corresponding Hamiltonian of the system. The commutation relations between ladder operators of different types are model dependent. The appropriate taking into account of them gives rise to different nonlinearly extended versions of the superconformal $\mathfrak{o} \mathfrak{g} \mathfrak{p}(2 \mid 2)$ symmetry [52].

It is interesting to note here that reflectionless (soliton and multisoliton) quantum systems can be constructed by applying DC transformations to the free quantum particle. The latter system is peculiar in virtue of the presence in it of additional integral of motion being the momentum operator that distinguishes the left- and right-moving plane wave eigenstates corresponding to the doubly degenerate energy levels of the free particle. In our case as the analog of the free particle we have the half-harmonic oscillator system $L_{0}$ characterized, as the harmonic oscillator, by the equidistant spectrum and by the pair of the conjugate second order ladder operators associated with such a peculiar nature of the spectrum. Any reflectionless system can be related with the free particle by two different intertwining operators whose action, however, does not produce any mutual displacement. A similar picture with two different sets of the intertwining operators acting without a relative shift appears also in the isospectral pairs of periodic finite-gap systems. Because of the absence of the relative displacement in the action of different intertwining operators in reflectionless and finite-gap systems, the analogs of our operators $\mathcal{C}^{ \pm}$built there by gluing different intertwining operators are not the ladder-type operators. Instead, they are the Lax-Novikov integrals of motion of the corresponding quantum systems related to the Korteweg-de Vries equation and its hierarchy; for the details see Refs. [53-57]. Moreover, in this context we still have another interesting analogy. One can consider a particular class of the quantum reflectionless systems given by the shape-invariant family of hyperbolic Pöschl-Teller systems described by the potentials of the form $-m(m+1) / \cosh ^{2} x$ [55]. Their reflectionless properties together with the presence of a nontrivial Lax-Novikov integral in each such system are explained naturally in terms of their DC relationship with the free particle corresponding to the case $m=0$. If we change in the indicated potential the integer parameter $m$ for real parameter $\nu$, we lose the connection with the free particle together with the reflectionless properties of the system. This analogy explains the peculiar nature of the rationally deformed conformal mechanics systems characterized by integer parameter $m$ in comparison with the general (anyonic) case with $g=\nu(\nu+1), \nu>-1 / 2$, $\nu \notin \mathbb{Z}$. For the latter class of the systems it is impossible to assemble the ladder operators of the types $\mathcal{A}^{ \pm}$and $\mathcal{C}^{ \pm}$, at least by the methods used here. Therefore, the problem of construction of the complete sets of the spectrum-generating ladder operators for such systems is open.

We saw that the neighbor pairs of the isotonic oscillator systems can be organized into the extended two-component systems that are described by the $\mathcal{N}=2$ supersymmetry. Such an extension can be realized in two different ways based on physical (positive) or nonphysical (negative) eigenstates of the corresponding Hamiltonians of the subsystems. In correspondence with this, in the first case we have the unbroken $\mathcal{N}=2$ supersymmetry, whereas in the second case the extended system is described by the broken supersymmetry. The interesting peculiarity we observed is that the products of the supercharges from the extended systems with unbroken and broken supersymmetries produce the ladder operators of the conformal mechanics systems. A similar observation was used recently in [58] to explain the origin of the hidden superconformal symmetry of the quantum harmonic oscillator.

It would be interesting to investigate coherent states in the rationally extended quantum harmonic oscillator systems as well as in the rational deformations of the conformal mechanics considered here. The appearance of some peculiarities can be expected in such systems due to the presence in them of different sets of the ladder operators.

It also seems to be very interesting to identify some class of quantum physical systems in which the presence of more than one pair of the ladder operators would be essential. One such possibility could correspond to the problems related to quantum transitions. 


\section{ACKNOWLEDGMENTS}

L. I. acknowledges the CONICYT scholarship 21170053. J. F. C. and M. S. P. acknowledge support from research projects FONDECYT 1130017 (Chile), Proyecto USA1555 Convenio Marco Universidades del Estado (Chile), MTM2015-64166-C2-1 (MINECO, Madrid), and DGA E24/1 (DGA, Zaragoza). J. F. C. thanks Universidad de Santiago de Chile for the kind hospitality. M. S.P. is grateful for the warm hospitality at Zaragoza University.

\section{APPENDIX A: DARBOUX TRANSFORMATIONS}

Here we briefly summarize a general scheme of the Darboux transformations and some of their properties. Let $L_{-}=-\frac{d^{2}}{d x^{2}}+V_{-}(x)$ be a Schrödinger operator and $\psi_{*}(x)$ be its eigenfunction, $L_{-} \psi_{*}=E_{*} \psi_{*}$. We do not worry about the singular or nonsingular nature of $L_{-}$and the physical or nonphysical nature of $\psi_{*}(x)$, and we generate the first order differential operators

$$
\begin{aligned}
& A_{\psi_{*}}^{-}=\psi_{*} \frac{d}{d x} \frac{1}{\psi_{*}}=\frac{d}{d x}+\mathcal{W}, \\
& A_{\psi_{*}}^{+}=-\frac{1}{\psi_{*}} \frac{d}{d x} \psi_{*}=-\frac{d}{d x}+\mathcal{W},
\end{aligned}
$$

where $\mathcal{W}=-\frac{\psi_{*}^{\prime}}{\psi_{*}}$. The operator $A_{\psi_{*}}^{+}$is the formal adjoint of $A_{\psi_{*}}^{-}$, and $\operatorname{ker} A_{\psi_{*}}^{-}=\psi_{*}, \operatorname{ker} A_{\psi_{*}}^{+}=1 / \psi_{*}$. We have a factorization relation $A_{\psi_{*}}^{+} A_{\psi_{*}}^{-}=L_{-}-E_{*}$, and a representation of the potential $V(x)$ in terms of the superpotential $\mathcal{W}$, $V_{-}=\mathcal{W}^{2}-\mathcal{W}^{\prime}$. The alternate product $A_{\psi_{*}}^{-} A_{\psi_{*}}^{+}=L_{+}-E_{*}$ defines the associated Schrödinger operator $L_{+}=-\frac{d^{2}}{d x^{2}}+$ $V_{+}$with the potential

$V_{+}=\mathcal{W}^{2}+\mathcal{W}^{\prime}=V_{-}+2 \mathcal{W}^{\prime}=V_{-}-2\left(\ln \psi_{*}\right)^{\prime \prime}$,

and $L_{+}\left(\psi_{*}\right)^{-1}=E_{*}\left(\psi_{*}\right)^{-1}$. With dependence on the nature of the wave function $\psi_{*}$, the so-obtained operator $L_{+}$can be singular or nonsingular, and the wave function $\left(\psi_{*}\right)^{-1}$ can describe a physical state or not. The operators $A_{\psi_{*}}^{-}$and $A_{\psi_{*}}^{+}$intertwine the Schrödinger operators $L_{-}$and $L_{+}$, $A_{\psi_{*}}^{-} L_{-}=L_{+} A_{\psi_{*}}^{-}, A_{\psi_{*}}^{+} L_{+}=L_{-} A_{\psi_{*}}^{+}$, and provide us with the mapping between their physical and nonphysical eigenfunctions. Namely, if $E \neq E_{*}$ and $\psi_{-, E}$ is an eigenfunction of $L_{-}, L_{-} \psi_{-, E}=E \psi_{-, E}$, then the function $A_{\psi_{*}}^{-} \psi_{-, E}$ is an eigenfunction of $L_{+}$of the same eigenvalue $E$. On the other hand, if $\psi_{+, E}$ is an eigenfunction of $L_{+}$, $L_{+} \psi_{+, E}=E \psi_{+, E}$, and $E \neq E_{*}$, then $A_{\psi_{*}^{+}}^{+} \psi_{+, E}$ is an eigenfunction of $L_{-}$of the same eigenvalue. In the case of $E=E_{*}$ we have the relations $A_{\psi_{*}}^{-} \psi_{*}=0, A_{\psi_{*}}^{+} \frac{1}{\psi_{*}}=0$, and

$$
\begin{aligned}
A_{\psi_{*}}^{-\widetilde{\psi_{*}}} & =\left(\psi_{*}\right)^{-1}, A_{\psi_{*}}^{+}\left(\widetilde{\left.\psi_{*}\right)^{-1}}=\psi_{*},\right. \\
\widetilde{\psi(x)} & =\psi(x) \int^{x} \frac{d \xi}{(\psi(\xi))^{2}} .
\end{aligned}
$$

Here $\widetilde{\psi(x)}$ is an eigenfunction linearly independent from a solution $\psi(x)$ of the stationary Schrödinger equation $L \psi=E \psi$. So, $\widetilde{\psi_{*}}$ in (A3) is the eigenfunction of eigenvalue $E_{*}$ of $L_{-}$to be linearly independent from $\psi_{*}$, and $\left(\widetilde{\left.\psi_{*}\right)^{-1}}\right.$ is the eigenfunction of the same eigenvalue $E_{*}$ of $L_{+}$that is linearly independent from its eigenfunction $\left(\psi_{*}\right)^{-1}$.

One can apply iteratively various Darboux transformations to produce a new system in $n$ steps. Such an $n$-step process of construction of a final system can be presented equivalently as a one-step DC transformation [41]. The eigenstates $\psi_{[n], \lambda}$ of the final system are obtained by mapping the eigenstates $\psi_{\lambda}$ of the original system and can be presented in two equivalent forms:

$$
\psi_{[n], \lambda}=\frac{W\left(\psi_{1}, \psi_{2}, \ldots, \psi_{n}, \psi_{\lambda}\right)}{W\left(\psi_{1}, \psi_{2}, \ldots, \psi_{n}\right)}=\mathbb{A}_{n}^{-} \psi_{\lambda} .
$$

A differential operator $\mathbb{A}_{n}^{-}$of order $n$ is constructed iteratively,

$$
\mathbb{A}_{n}^{-}=A_{n}^{-} A_{n-1}^{-} \cdots A_{1}^{-}, \quad A_{i}^{-}=\mathbb{A}_{i-1}^{-} \psi_{i} \frac{d}{d x} \frac{1}{\mathbb{A}_{i-1}^{-} \psi_{i}},
$$

where $i=1, \ldots, n$, and $A_{0}^{-}=1$. This operator intertwines the Hamiltonian of the initial system directly with the Hamiltonian of the final system. Function (A4) is a solution of the Schrödinger eigenvalue problem

$$
\left(-\frac{d^{2}}{d x^{2}}+V_{[n]}(x)\right) \psi_{[n], \lambda}(x)=E_{\lambda} \psi_{[n], \lambda}(x),
$$

where $V_{[n]}=V-2\left(\ln W\left(\psi_{1}, \ldots, \psi_{n}\right)\right)^{\prime \prime}$ is a potential of the corresponding final system. The DC transformation is defined by the choice of the set of eigenfunctions $\left(\psi_{1}, \psi_{2}, \ldots, \psi_{n}\right)$ of the initial system $L=-\frac{d^{2}}{d x^{2}}+V(x)$, and we refer to the corresponding transformation as a $\left(\psi_{1}, \psi_{2}, \ldots, \psi_{n}\right)$ scheme. The change in the chosen order of the eigenfunctions $\psi_{i}$ generates different intermediate operators $\mathbb{A}_{i}^{-}$and $A_{i}^{-}$in factorization (A5), but has no effect on the $n$th order differential operator $\mathbb{A}_{n}^{-}$and the potential $V_{[n]}$. Nevertheless, we see that the order in which the seed states are taken is not unique, and the intermediate schemes that occur in the $n$th order DC transformation depend on the order in the chosen set of eigenfunctions of the Hamiltonian operator of the initial system. The kernel of $\mathbb{A}_{n}^{-}$is spanned by the chosen set of the seed states, $\operatorname{ker} \mathbb{A}_{n}^{-}=\operatorname{span}\left\{\psi_{1}, \psi_{2}, \ldots, \psi_{n}\right\}$, while $\operatorname{ker} \mathbb{A}_{n}^{+}=$ $\operatorname{span}\left\{\mathbb{A}_{n}^{-} \widetilde{\psi_{1}}, \mathbb{A}_{n}^{-} \widetilde{\psi_{2}}, \ldots, \mathbb{A}_{n}^{-} \widetilde{\psi_{n}}\right\}$. Equation (A4) can be presented in an equivalent form

$$
\begin{aligned}
\psi_{[n], \lambda} & =\left(A_{n}^{-} A_{n-1}^{-} \cdots A_{2}^{-}\right)\left(A_{1}^{-} \psi_{\lambda}\right) \\
& =\frac{W\left(A_{1}^{-} \psi_{2}, \ldots, A_{1}^{-} \psi_{n}, A_{1}^{-} \psi_{\lambda}\right)}{W\left(A_{1}^{-} \psi_{2}, \ldots, A_{1}^{-} \psi_{n}\right)} .
\end{aligned}
$$


Combining Eqs. (A4) and (A7), we obtain a new expression for Wronskian,

$$
\begin{aligned}
& W\left(\psi_{1}, \psi_{2}, \ldots, \psi_{n}, \psi_{\lambda}\right) \\
& \quad=W\left(\psi_{1}, \psi_{2}, \ldots, \psi_{n}\right) \frac{W\left(A_{1}^{-} \psi_{2}, \ldots, A_{1}^{-} \psi_{n}, A_{1}^{-} \psi_{\lambda}\right)}{W\left(A_{1}^{-} \psi_{2}, \ldots, A_{1}^{-} \psi_{n}\right)} .
\end{aligned}
$$

Consider now the scheme $\left(\psi_{1}, \widetilde{\psi_{1}}, \psi_{2}, \ldots, \psi_{n}\right)$. We realize the first step on the basis of the function $\psi_{1}$ that produces the intertwining operator $A_{1}^{-}$. Its action on $\widetilde{\psi_{1}}$ generates the function $A_{1}^{-} \widetilde{\psi_{1}}=1 / \psi_{1}$; see Eq. (A3). According to (A5) and (A1), we obtain then $A_{2}^{-}=-\left(A_{1}^{-}\right)^{\dagger}$. When applied twice, Eq. (A7) in this case gives us relations

$$
\frac{W\left(\psi_{1}, \widetilde{\psi}_{1}, \psi_{2}, \ldots, \psi_{n}, \psi_{\lambda}\right)}{W\left(\psi_{1}, \widetilde{\psi}_{1}, \psi_{2}, \ldots, \psi_{n}\right)}=\frac{W\left(A_{1}^{\dagger} A_{1}^{-} \psi_{2}, \ldots, A_{1}^{\dagger} A_{1}^{-} \psi_{n}, A_{1}^{\dagger} A_{1}^{-} \psi_{\lambda}\right)}{W\left(A_{1}^{\dagger} A_{1}^{-} \psi_{2}, \ldots, A_{1}^{\dagger} A_{1}^{-} \psi_{n}\right)}=\frac{W\left(\psi_{2}, \ldots, \psi_{n}, \psi_{\lambda}\right)}{W\left(\psi_{2}, \ldots, \psi_{n}\right)}
$$

Here we used the fact that the second order operator $A_{1}^{\dagger} A_{1}^{-}$ is equal to the original Hamiltonian shifted for some constant, and $\psi_{i}, i=2, \ldots, n$, are its eigenfunctions. The second equality in (A9) is modulo some nonzero constant multiplier. Note that for a scheme $\left(\psi_{1}, \widetilde{\psi_{1}}, \psi_{2}\right)$, by (A4) we obtain the relation $W\left(\psi_{1}, \widetilde{\psi_{1}}, \psi_{2}\right)=W\left(\psi_{1}, \widetilde{\psi_{1}}\right) A_{1}^{\dagger} A_{1}^{-} \psi_{2}$. Since $W\left(\psi_{1}, \widetilde{\psi_{1}}\right)=$ const $\neq 0$, the equality reduces to $W\left(\psi_{1}, \widetilde{\psi}_{1}, \psi_{2}\right)=C \psi_{2}$. This corresponds to a particularly simple case of a relation $W\left(\psi_{2}, \ldots, \psi_{n}, \psi_{\lambda}\right)=$ $W\left(\psi_{1}, \widetilde{\psi_{1}}, \psi_{2}, \ldots, \psi_{n}, \psi_{\lambda}\right)$ directly following from (A9).

\section{APPENDIX B: EQUIVALENT REPRESENTATIONS FOR EIGENFUNCTIONS OF $L_{m}$}

Equation (2.13) can be used to establish the relationship between eigenfunctions (2.11) of the isotonic oscillator given in terms of the Hermite polynomials and their standard representation in terms of the generalized Laguerre polynomials.

The normalized form of the eigenstates (2.11) is given by the relation $\hat{\psi}_{m, l}=h_{m, l} \psi_{m, l}$ with normalization coefficients $h_{m, l}=2^{-(2 m+l)} \pi^{-1 / 4}((m+l) !(4 m+4 l+1) ! / l !)^{-1 / 2}$. On the other hand, the $l$ th normalized eigenstate of the isotonic oscillator (2.2) with $g=m(m+1)$ is given by the relation [4] $\hat{\psi}_{m, l}=\left(2 \cdot l ! / \Gamma\left(m+l+\frac{3}{2}\right)\right)^{1 / 2}(-1)^{l} x^{m+1} \mathcal{L}_{l}^{m+1}\left(x^{2}\right)$, and we obtain the equality

$$
\begin{aligned}
\mathbb{A}_{m}^{-}\left(H_{2 n+1}(x) e^{-x^{2} / 2}\right) & =C_{n m} x^{m+1} \mathcal{L}_{n-m}^{(m+1 / 2)}\left(x^{2}\right) e^{-x^{2} / 2}, \\
C_{n m} & =(-1)^{n+m} 2^{2 n+m+1} n !,
\end{aligned}
$$

where $n=m+l \geq m$. Equation (B1) can be extended for the case $m=0$ if we put here $\mathbb{A}_{0}=1$ in correspondence with definition (A5). Multiplying this relation by $e^{x^{2} / 2}$ from the left, we obtain

$$
\begin{aligned}
x^{m+1} \mathcal{L}_{n-m}^{(m+1 / 2)}\left(x^{2}\right) & =C_{n m}^{-1} \mathcal{D}_{m} H_{2 n+1}(x), \\
\mathcal{D}_{m} & =\left(\frac{d}{d x}-\frac{m}{x}\right) \cdots\left(\frac{d}{d x}-\frac{1}{x}\right) .
\end{aligned}
$$

Note that the differential operator $\mathcal{D}_{m}$ of order $m$ appearing in (B2) has exactly the form of the operator that intertwines the Hamiltonian operator of a free quantum particle on the positive half-line and that of the Calogero model with coupling constant $m(m+1)$ [46]. One can invert relation (B2) by multiplying it from the left by the operator $e^{x^{2} / 2} \mathbb{A}_{m}^{+}$ and applying Eq. (2.13). This gives the relation

$$
\begin{aligned}
H_{2 n+1}(x)= & (-1)^{n+m}(n-m) ! 2^{2 n+m+1} \\
& \times \mathcal{D}_{m}^{\dagger}\left(x^{m+1} \mathcal{L}_{n-m}^{(m+1 / 2)}\left(x^{2}\right)\right) .
\end{aligned}
$$

Differentiating (B3) and using the relation $\left(H_{n}(x)\right)^{\prime}=$ $2 n H_{n-1}(x)$ we also obtain the relation

$$
\begin{aligned}
H_{2 n}(x)= & (-1)^{n+m}(n-m) ! 2^{2 n+m}(2 n+1)^{-1} \frac{d}{d x} \\
& \times \mathcal{D}_{m}^{\dagger}\left(x^{m+1} \mathcal{L}_{n-m}^{(m+1 / 2)}\left(x^{2}\right)\right) .
\end{aligned}
$$

Equations (B3) and (B4) generalize the well known relations of the case $m=0$ with $\mathcal{D}_{0}=\mathcal{D}_{0}^{\dagger}=1$ between the generalized Laguerre and Hermite polynomials: $H_{2 n+1}(x)=(-1)^{n} 2^{2 n} n ! 2 x \mathcal{L}_{n}^{(1 / 2)}\left(x^{2}\right) \quad$ and $\quad H_{2 n}(x)=$ $(-1)^{n} 2^{2 n} n ! \mathcal{L}_{n}^{(-1 / 2)}\left(x^{2}\right)$, where for obtaining the last equality we also used the relation $\frac{d}{d x}\left(x^{\alpha} \mathcal{L}_{n}^{(\alpha)}(x)\right)=$ $(n+\alpha) x^{\alpha-1} \mathcal{L}_{n}^{(\alpha-1)}(x)$ with $\alpha=1 / 2$.

\section{APPENDIX C: PROOF OF RELATION (4.1)}

Our goal here is to prove relation (4.1), for which the key point will be the equality $H_{2 n+1}(x)=x \mathcal{L}_{n}^{(1 / 2)}\left(x^{2}\right)$ modulo inessential numerical constant. Consider the Wronskian identities $W\left(u(x) h_{1}(x), \ldots, u(x) h_{m}(x)\right)=$ $u^{m}(x) W\left(h_{1}(x), \ldots, h_{m}(x)\right)$, where $h_{n}(x), \quad n=1, \ldots, m$, and $u(x)$ are some arbitrary functions of $x$, and $W\left(h_{1}\left(x^{2}\right), \ldots, h_{m}\left(x^{2}\right)\right)=\left.x^{m(m-1) / 2} W\left(h_{1}(z), \ldots, h_{m}(z)\right)\right|_{z=x^{2}}$. Then for an arbitrary set of odd states of the harmonic oscillator we have 


$$
W\left(\psi_{2 n_{1}+1}, \ldots, \psi_{2 m_{1}+1}\right)=x^{m(m+1) / 2} e^{-m x^{2} / 2} g_{\left(-\left(2 n_{1}+1\right),-\left(2 n_{2}+1\right), \ldots,-\left(2 n_{m}+1\right)\right)}(x),
$$

where $g_{\left(-\left(2 n_{1}+1\right),-\left(2 n_{2}+1\right), \ldots,-\left(2 n_{m}+1\right)\right)}(x)=\left.W\left(\mathcal{L}_{n_{1}}^{(1 / 2)}(z), \ldots, \mathcal{L}_{n_{m}}^{(1 / 2)}(z)\right)\right|_{z=x^{2}}$. Using the derivative relation for the generalized Laguerre polynomial, $\frac{d^{k}}{d z^{k}}\left(\mathcal{L}_{n}^{(\alpha)}(z)\right)=(-1)^{k} \mathcal{L}_{n-k}^{(\alpha+k)}(z)$ if $k<n$; otherwise $\frac{d^{k}}{d z^{k}}\left(\mathcal{L}_{n}^{(\alpha)}\right)(z)=0$, and also the relation $\mathcal{L}_{0}^{(\alpha)}=1$, for a particular case when $n_{j}=j, j=0,1, \ldots, m-1$, one finds that the function $g_{(1, \ldots, 2 m-1)}$ is a determinant of some triangular matrix with constant numbers in the diagonal, and then $W(1, \ldots, 2 m-1)=C x^{m(m+1) / 2} e^{-x^{2} / 2}$. Under the transformation $x \rightarrow i x$ the Wronskian (C1) takes the form

$$
W\left(\psi_{2 n_{1}+1}^{-}, \psi_{2 n_{2}+1}^{-}, \ldots, \psi_{2 n_{m}+1}^{-}\right)=e^{m x^{2} / 2} x^{m(m+1) / 2} f_{\left(-\left(2 n_{1}+1\right),-\left(2 n_{2}+1\right), \ldots,-\left(2 n_{m}+1\right)\right)}(x)
$$

with $f_{\left(-\left(2 n_{1}+1\right), \ldots,-\left(2 n_{m}+1\right)\right)}(x)=g_{\left(\left(2 n_{1}+1\right), \ldots,\left(2 n_{m}+1\right)\right)}(i x)$. This is just a particular example of a nonsingular Wronskian of nonphysical eigenstates.

\section{APPENDIX D: LIST OF SOME DEFORMED CONFORMAL MECHANICS POTENTIALS}

Here we display the explicit form of some deformations of the conformal mechanics potentials $V_{m}=x^{2}+\frac{m(m+1)}{x^{2}}$ discussed in the main part of the text:

$$
\begin{gathered}
V_{(-3,-7)}=V_{2}-4+24 \frac{32 x^{10}+240 x^{8}+528 x^{6}-840 x^{4}-3654 x^{2}-2205}{\left(8 x^{6}+60 x^{4}+126 x^{2}+105\right)^{2}}, \\
V_{(1,4,5)}=V_{1}+6+8 \frac{96 x^{10}-48 x^{8}-144 x^{6}-920 x^{4}+230 x^{2}-75}{\left(8 x^{6}-4 x^{4}+10 x^{2}+15\right)^{2}}, \\
V_{(1,5,6)}=V_{1}+6+16 \frac{256 x^{14}-960 x^{12}+1152 x^{10}-5712 x^{8}+432 x^{6}+9180 x^{4}-3960 x^{2}-675}{\left(16 x^{8}-48 x^{6}+72 x^{4}+60 x^{2}+45\right)^{2}}, \\
V_{(1,4,5,10,11)}=10+V_{1}+16 \frac{N(x)}{D(x)},
\end{gathered}
$$

where

$$
\begin{aligned}
N(x)= & -72937816875+359826563250 x^{2}-3559365463500 x^{4}+1124647108200 x^{6} \\
& +368202542400 x^{8}+1343539612800 x^{10}-1951252934400 x^{12}+822933619200 x^{14} \\
& -1455591836160 x^{16}+1118053063680 x^{18}-1055756298240 x^{20} \\
& +535377653760 x^{22}-147987087360 x^{24}+32552681472 x^{26}-9212461056 x^{28} \\
& +5052694528 x^{30}-1919090688 x^{32}+402784256 x^{34}-49020928 x^{36}+2621440 x^{38} \\
D(x)= & \left(467775+623700 x^{2}-374220 x^{4}+1995840 x^{6}-702240 x^{8}+94080 x^{10}\right. \\
& \left.+146560 x^{12}-64512 x^{14}+45824 x^{16}-11264 x^{18}+1024 x^{20}\right)^{2} .
\end{aligned}
$$

[1] V. de Alfaro, S. Fubini, and G. Furlan, Conformal invariance in quantum mechanics, Nuovo Cimento Soc. Ital. Fis. A 34, 569 (1976).

[2] F. Calogero, Solution of a three-body problem in onedimension, J. Math. Phys. (N.Y.) 10, 2191 (1969).

[3] F. Calogero, Solution of the one-dimensional $N$ body problems with quadratic and/or inversely quadratic pair potentials, J. Math. Phys. (N.Y.) 12, 419 (1971).
[4] A. M. Perelomov, Algebraic approach to the solution of a one-dimensional model of $N$ interacting particles, Theor. Math. Phys. 6, 263 (1971).

[5] Y. Weissman and J. Jortner, The isotonic oscillator, Phys. Lett. 70A, 177 (1979).

[6] J. M. Leinaas and J. Myrheim, On the theory of identical particles, Nuovo Cimento Soc. Ital. Fis. B 37, 1 (1977). 
[7] R. MacKenzie and F. Wilczek, Peculiar spin and statistics in two space dimensions, Int. J. Mod. Phys. A 03, 2827 (1988).

[8] A. P. Polychronakos, Physics and mathematics of Calogero particles, J. Phys. A 39, 12793 (2006).

[9] V. P. Akulov and A. I. Pashnev, Quantum superconformal model in $(1,2)$ space, Theor. Math. Phys. 56, 862 (1983).

[10] S. Fubini and E. Rabinovici, Superconformal quantum mechanics, Nucl. Phys. B245, 17 (1984).

[11] E. A. Ivanov, S. O. Krivonos, and V. M. Leviant, Geometry of conformal mechanics, J. Phys. A 22, 345 (1989).

[12] E. A. Ivanov, S. O. Krivonos, and V. M. Leviant, Geometric superfield approach to superconformal mechanics, J. Phys. A 22, 4201 (1989).

[13] D.Z. Freedman and P. F. Mende, An exactly solvable $N$ particle system in supersymmetric quantum mechanics, Nucl. Phys. B344, 317 (1990).

[14] N. Wyllard, (Super)conformal many body quantum mechanics with extended supersymmetry, J. Math. Phys. (N.Y.) 41, 2826 (2000).

[15] M. Asorey, J. F. Cariñena, G. Marmo, and A. Perelomov, Isoperiodic classical systems and their quantum counterparts, Ann. Phys. (Amsterdam) 322, 1444 (2007).

[16] J. F. Cariñena, A. M. Perelomov, and M. F. Rañada, Isochronous classical systems and quantum systems with equally spaced spectra, J. Phys. Conf. Ser. 87, 012007 (2007).

[17] J. M. Maldacena, The large $N$ limit of superconformal field theories and supergravity, Int. J. Theor. Phys. 38, 1113 (1999); [Adv. Theor. Math. Phys. 2, 231 (1998)].

[18] S. S. Gubser, I. R. Klebanov, and A. M. Polyakov, Gauge theory correlators from noncritical string theory, Phys. Lett. B 428, 105 (1998).

[19] E. Witten, Anti-de Sitter space and holography, Adv. Theor. Math. Phys. 2, 253 (1998).

[20] P. Claus, M. Derix, R. Kallosh, J. Kumar, P. K. Townsend, and A. Van Proeyen, Black Holes and Superconformal Mechanics, Phys. Rev. Lett. 81, 4553 (1998).

[21] J. A. de Azcarraga, J. M. Izquierdo, J. C. Perez Bueno, and P. K. Townsend, Superconformal mechanics and nonlinear realizations, Phys. Rev. D 59, 084015 (1999).

[22] G. W. Gibbons and P. K. Townsend, Black holes and Calogero models, Phys. Lett. B 454, 187 (1999).

[23] J. Michelson and A. Strominger, Superconformal multiblack hole quantum mechanics, J. High Energy Phys. 09 (1999) 005.

[24] R. Britto-Pacumio, J. Michelson, A. Strominger, and A. Volovich, Lectures on superconformal quantum mechanics and multi-black hole moduli spaces, NATO Sci. Ser. C 556, 255 (2000).

[25] G. Papadopoulos, Conformal and superconformal mechanics, Classical Quantum Gravity 17, 3715 (2000).

[26] B. Pioline and A. Waldron, Quantum Cosmology and Conformal Invariance, Phys. Rev. Lett. 90, 031302 (2003).

[27] S. Fedoruk, E. Ivanov, and O. Lechtenfeld, Superconformal mechanics, J. Phys. A 45, 173001 (2012).

[28] J. Gonera, Conformal mechanics, Ann. Phys. (Amsterdam) 335, 61 (2013).

[29] G. F. de Teramond, H. G. Dosch, and S. J. Brodsky, Baryon spectrum from superconformal quantum mechanics and its light-front holographic embedding, Phys. Rev. D 91, 045040 (2015).

[30] S. J. Brodsky, G. F. de Téramond, H. G. Dosch, and C. Lorcé, Universal effective hadron dynamics from superconformal algebra, Phys. Lett. B 759, 171 (2016).

[31] S. Yu. Dubov, V. M. Eleonskii, and N. E. Kulagin, Equidistant spectra of anharmonic oscillators, Zh. Eksp. Teor. Fiz. 102, 814 (1992).

[32] J. F. Cariñena, A. M. Perelomov, M. F. Rañada, and M. Santander, A quantum exactly solvable nonlinear oscillator related to the isotonic oscillator, J. Phys. A 41, 085301 (2008).

[33] J. M. Fellows and R. A. Smith, Factorization solution of a family of quantum nonlinear oscillators, J. Phys. A 42, 335303 (2009).

[34] D. Gómez-Ullate, N. Kamran, and R. Milson, An extension of Bochner's problem: Exceptional invariant subspaces, J. Approx. Theory 162, 987 (2010).

[35] C. Quesne, Exceptional orthogonal polynomials, exactly solvable potentials and supersymmetry, J. Phys. A 41, 392001 (2008).

[36] Y. Grandati, Solvable rational extensions of the isotonic oscillator, Ann. Phys. (Amsterdam) 326, 2074 (2011).

[37] I. Marquette, New families of superintegrable systems from $k$-step rational extensions, polynomial algebras and degeneracies, J. Phys. Conf. Ser. 597, 012057 (2015).

[38] J. F. Cariñena and M. S. Plyushchay, Ground-state isolation and discrete flows in a rationally extended quantum harmonic oscillator, Phys. Rev. D 94, 105022 (2016).

[39] G. Darboux, Sur une proposition relative aux équations linéaires, C.R. Hebd. Seances Acad. Sci. 94, 1456 (1882).

[40] M. M. Crum, Associated Sturm-Liouville systems, Quart. J. Math. Oxford 6, 121 (1955).

[41] V. B. Matveev and M. A. Salle, Darboux Transformations and Solitons (Springer, Berlin, 1991).

[42] M. G. Krein, On a continuous analogue of a Christoffel formula from the theory of orthogonal polynomials, Dokl. Akad. Nauk SSSR 113, 970 (1957).

[43] V. E. Adler, A modification of Crum's method, Theor. Math. Phys. 101, 1381 (1994).

[44] A. Oblomkov, Monodromy-free Schrödinger operators with quadratically increasing potentials, Theor. Math. Phys. 121, 1574 (1999).

[45] J. F. Cariñena and M.S. Plyushchay, ABC of ladder operators for rationally extended quantum harmonic oscillator systems, J. Phys. A 50, 275202 (2017).

[46] J. M. Guilarte and M.S. Plyushchay, Perfectly invisible $\mathcal{P} \mathcal{T}$-symmetric zero-gap systems, conformal field theoretical kinks, and exotic nonlinear supersymmetry, J. High Energy Phys. 12 (2017) 061.

[47] H. Airault, H. P. McKean, and J. Moser, Rational and elliptic solutions of the Korteweg-de Vries equation and a related many-body problem, Commun. Pure Appl. Math. 30, 95 (1977).

[48] M. Adler and J. Moser, On a class of polynomials connected with the Korteweg-deVries equation, Commun. Math. Phys. 61, 1 (1978).

[49] A. Gorsky and N. Nekrasov, Hamiltonian systems of Calogero type and two-dimensional Yang-Mills theory, Nucl. Phys. B414, 213 (1994). 
[50] J. F. Cariñena, G. Marmo, A. M. Perelomov, and M. F. Rañada, Related operators and exact solutions of Schrödinger equations, Int. J. Mod. Phys. 13, 4913 (1998).

[51] J. de Boer, F. Harmsze, and T. Tjin, Nonlinear finite $W$ symmetries and applications in elementary systems, Phys. Rep. 272, 139 (1996).

[52] L. Inzunza and M. S. Plyushchay (in preparation).

[53] F. Correa, V. Jakubsky, L. M. Nieto, and M. S. Plyushchay, Self-Isospectrality, Special Supersymmetry, and Their Effect on the Band Structure, Phys. Rev. Lett. 101, 030403 (2008).

[54] F. Correa, V. Jakubsky, and M. S. Plyushchay, Finite-gap systems, tri-supersymmetry and self-isospectrality, J. Phys. A 41, 485303 (2008).
[55] F. Correa, V. Jakubsky, and M. S. Plyushchay, AharonovBohm effect on $\operatorname{AdS}(2)$ and nonlinear supersymmetry of reflectionless Poschl-Teller system, Ann. Phys. (Amsterdam) 324, 1078 (2009).

[56] A. Arancibia, J. M. Guilarte, and M. S. Plyushchay, Effect of scalings and translations on the supersymmetric quantum mechanical structure of soliton systems, Phys. Rev. D 87, 045009 (2013).

[57] A. Arancibia, J. M. Guilarte, and M. S. Plyushchay, Fermion in a multi-kink-antikink soliton background, and exotic supersymmetry, Phys. Rev. D 88, 085034 (2013).

[58] L. Inzunza and M. S. Plyushchay, Hidden superconformal symmetry: Where does it come from?, Phys. Rev. D 97, 045002 (2018). 